\title{
Localisation of extended brain sources from EEG/MEG: the ExSo-MUSIC approach
}

\author{
Gwénaël Birot ${ }^{\mathrm{a}, \mathrm{b}}$, Laurent Albera ${ }^{\mathrm{a}, \mathrm{b}}$, Fabrice Wendling, ${ }^{\mathrm{a}, \mathrm{b}}$, Isabelle Merlet ${ }^{\mathrm{a}, \mathrm{b}, *}$ \\ ${ }^{a}$ Inserm - UMR 642, Campus de Beaulieu - Bat. 22, Rennes, F-35042, France \\ ${ }^{b}$ Université de Rennes 1, LTSI, Campus de Beaulieu-Bat. 22, Rennes, F-35042, France
}

\section{Abstract}

We propose a new MUSIC-like method, called 2q-ExSo-MUSIC $(q \geq 1)$. This method is an extension of the $2 q$-MUSIC $(q \geq 1)$ approach for solving the EEG/MEG inverse problem, when spatially-extended neocortical sources ("ExSo") are considered. It introduces a novel ExSo-MUSIC principle. The novelty is two-fold: i) the parameterization of the spatial source distribution that leads to an appropriate metric in the context of distributed brain sources and ii) the introduction of an original, efficient and low-cost way of optimizing this metric. In 2q-ExSo-MUSIC, the possible use of higher order statistics $(q \geq 2)$ offers a better robustness with respect to Gaussian noise of unknown spatial coherence and modeling errors. As a result we reduced the penalizing effects of both the background cerebral activity that can be seen as a Gaussian and spatially correlated noise, and the modeling errors induced by the non-exact resolution of the forward problem. Computer results on

\footnotetext{
*Corresponding author: Tel: (33) - 223236220 , Fax (33) - 223236917

Email addresses: gwenael.birot@univ-rennes1.fr (Gwénaël Birot), laurent .albera@univ-rennes1.fr (Laurent Albera), fabrice.wendling@univ-rennes1.fr (Fabrice Wendling), isabelle.merlet@univ-rennes1.fr (Isabelle Merlet)
} 
simulated EEG signals obtained with physiologically-relevant models of both the sources and the volume conductor show a highly increased performance of our $2 q$-ExSo-MUSIC method as compared to the classical $2 q$-MUSIC algorithms.

Keywords: Source localization, distributed sources, EEG, MEG, MUSIC, higher order statistics.

\section{Introduction}

Since the 1980's, source localization methods based on advanced signal processing techniques have been proposed to identify the intracerebral generators underlying surface ElectroEncepghaloGraphic (EEG) or MagnetoEncephaloGraphic (MEG) signals. Particularly, in the context of drug resistant partial epilepsy, source localization methods have been widely applied to interictal spikes. Indeed, these paroxysms witness the patient's epilepsy and, compared to ictal discharges, appear in the large majority of cases on surface recordings with a higher Signal-to-Noise Ratio (SNR). For reviews on this topic, the reader may refer to (Ebersole, 2000; Funke et al., 2009; Merlet, 2001; Michel et al., 2004; Plummer et al., 2008; Verhellen and Boon, 2007).

In the large family of techniques aimed at solving the inverse problem, methods based on Minimum Norm Estimates (MNE) reconstruct the source activity at each location of the brain volume in a tomographic way. They are particularly suited for the localization of distributed generators of epileptic interictal spikes and their clinical yield was recently adressed (Plummer et al., 2010). While these methods proved to be very efficient under certain conditions, they suffer from certain drawbacks (see review in Grech et al., 
2008). In particular, their resolution is blurred when a $L_{2}$-norm of the dipole intensity gradient is used in order to regularize the inverse problem. Other approaches such as Second Order (SO) MUSIC-like algorithms (de Munck and Bijma, 2009; Mosher and Leahy, 1999; Mosher et al., 1992; Schmidt, 1986) search for the best dipoles by exploring the parameter space (location and orientation). These SO methods are very powerful in multiple sources environments under the three following hypotheses: i) the number of sources is less than the number of sensors, ii) the spatial coherence of the noise is known and iii) there is no errors in the forward model. Indeed, SO approaches infinite asymptotic precision and resolution whatever the Signal to Noise Ratio (SNR) (Kaveh and Barabell, 1986; Porat and Friedlander, 1988; Stoica and Nehorai, 1989, 1990). However, in the particular context of localizing epileptic cerebral sources, none of the three aforementioned assumptions holds. Indeed, i) the sources of EEG epileptic activity are usually distributed over a relatively wide cortical area that involves much more dipoles than the number of scalp electrodes, ii) the EEG background activity behaves at as a Gaussian noise of unknown spatial coherence, and iii) the forward model is corrupted by errors induced by a possible misplacement of electrodes, by incorrect conductivity values for the headmodel, and by the estimation of brain, skull and skin surfaces. Contrarily to SO MUSIC-like methods, HO approaches (Albera et al., 2008; Birot et al., 2010; Chevalier et al., 2006, 2007) are asymptotically insensitive to any Gaussian noise, since HO statistics of Gaussian random variables are zero (McCullagh, 1987). They are also shown to be much less sensitive to modelling errors since, from the virtual array theory, the use of HO statistics virtually increases the number of sen- 
sors. Moreover, HO methods are able to process more independent sources than the number of sensors but this still remains insufficient to localize all the possibly-correlated dipoles involved in epilepic distributed sources.

Under certain conditions (evoked potentials or epilepsy for instance), the spatial correlation within activated cortical areas can be exploited in order to summarize the high number of dipoles into a unique entity called Extended Source (ExSo) and consequently to return to an overdetermined mixture. This property is particularly appropriate in the context of epileptic events. Indeed, several studies showed that these events can be detected on surface EEG recordings because they are likely to involve large cortical areas, in a highly synchronized manner (Cosandier-Rimélé et al., 2008; Ebersole, 1997; Gavaret et al., 2006; Merlet and Gotman, 1999; Tao et al., 2005).

In this paper, we propose a new source localization method specifically adapted to such situations where the synchronization of dipole sources within activated areas is high. This novel MUSIC-like method, called $2 q$-ExSoMUSIC $(q \geq 1)$, uses a new principle for the localisation of ExSo's from EEG/MEG data (ExSo-MUSIC) and make use of SO or HO statistics. The ExSo-MUSIC principle i) take advantage of the synchronization of dipoles within each ExSo's and ii) uses a MUSIC-like criterion in order to search for several simple-shaped surfaces which reunion fits the actual ExSo's. The generic method is proposed at any even statistical order and therefore permits the use of higher orders when robustness with respect to the background activity and to modelling errors is needed. In order to quantitatively validate our method, we evaluated its performance at second and fourth order on simulated EEG epileptic spikes obtained with physiologically relevant models 
of both sources and volume conduction (Cosandier-Rimélé et al., 2007, 2008; Wendling et al., 2000) and compared it to the MUSIC algorithm as used in distributed brain source localization (Küçükaltun-Yildirim et al., 2006), and its extension to Fourth Order (FO).

\section{Materials and methods}

\subsection{Forward model and problem formulation}

It is commonly admitted that the signals recorded at the head surface mostly reflect the activity of pyramidal cells within the cortex. These activities can be modelled by current dipoles oriented orthogonally to the cortical surface. Consequently, in our study, the source space $\Omega$ is restricted to the cortical surface and the source distribution can be seen as a field of current dipoles over $\Omega$. Each of these dipoles is defined by its location $\rho \in \Omega$ and its electrical activity $s(\boldsymbol{\rho}, t)$. Under these considerations, the $N$-channelsEEG/MEG model used throughout this paper has the classical linear form

$$
\boldsymbol{x}(t)=\sum_{\boldsymbol{\rho} \in \Omega} \boldsymbol{a}(\boldsymbol{\rho}) s(\boldsymbol{\rho}, t)+\boldsymbol{\nu}_{i}(t)
$$

where $\{\boldsymbol{x}(t)\}$ is the EEG/MEG data, $\left\{\boldsymbol{\nu}_{i}(t)\right\}$ is a Gaussian instrument noise independent of dipole activity $\{s(\boldsymbol{\rho}, t)\}$. The noise due to artefacts (muscular, cardiac or ocular) is not represented here since specific techniques have been developed to remove it (Albera et al., 2010; Congedo et al., 2008; James and Hesse, 2005; Vigario and Oja, 2008), and denoising is outside the scope of this study. The $N$-dimensional known leadfield vector $\boldsymbol{a}(\boldsymbol{\rho})$ transcripts the contribution of a unitary dipole located at $\boldsymbol{\rho}$ and oriented orthogonally to the cortical surface to the set of sensors. 
Following the superposition principle, interictal epileptic spikes appearing on EEG data can be considered as the sum of i) epileptic activity rising from epileptic sources and ii) normal background activity distributed all over the source space (see figure 1(a)). Denoting by $\Theta$ the origin of epileptic activity, the EEG/MEG vector (1) can be split as

$$
\boldsymbol{x}(t)=\underbrace{\sum_{\boldsymbol{\rho} \in \Theta} \boldsymbol{a}(\boldsymbol{\rho}) s_{\mathrm{e}}(\boldsymbol{\rho}, t)}_{\text {signal due to epileptic activity }}+\underbrace{\sum_{\boldsymbol{\rho} \in \Omega} \boldsymbol{a}(\boldsymbol{\rho}) s_{\mathrm{b}}(\boldsymbol{\rho}, t)}_{\text {signal due to background activity }}+\boldsymbol{\nu}_{i}(t)
$$

where $s_{\mathrm{e}}(\boldsymbol{\rho}, t)$ is the epileptic activity at location $\boldsymbol{\rho}$, i.e. the signal of interest and $s_{\mathrm{b}}(\boldsymbol{\rho}, t)$ is the background activity at location $\boldsymbol{\rho}$, i.e. the signal of noninterest. The problem dealt with by the proposed $2 q$-ExSo-MUSIC method is the identification of the origin $\Theta$ of epileptic activity.

\subsection{Extended source}

The configuration of cerebral sources of EEG/MEG signals can be complex depending on space/time parameters under consideration. Nevertheless, is it well admitted that for EEG events in general, and epileptic spikes in particular, to be detectable on scalp EEG, a high synchronization level of intracerebral activity within a large area of cortex is necessary. The $2 q$-ExSoMUSIC is built upon this property irrespectively of the spatial configuration of sources. Consequently, an extended source or ExSo (used synonymously to "distributed" source) is defined in the following as a set of contiguous dipoles (i.e patches) exhibiting a highly synchronized activity. When the activity between two (or more) spatially distinct patches is also highly synchronized, then the presented 2q-ExSo-MUSIC method will consider it as a single ExSo. 

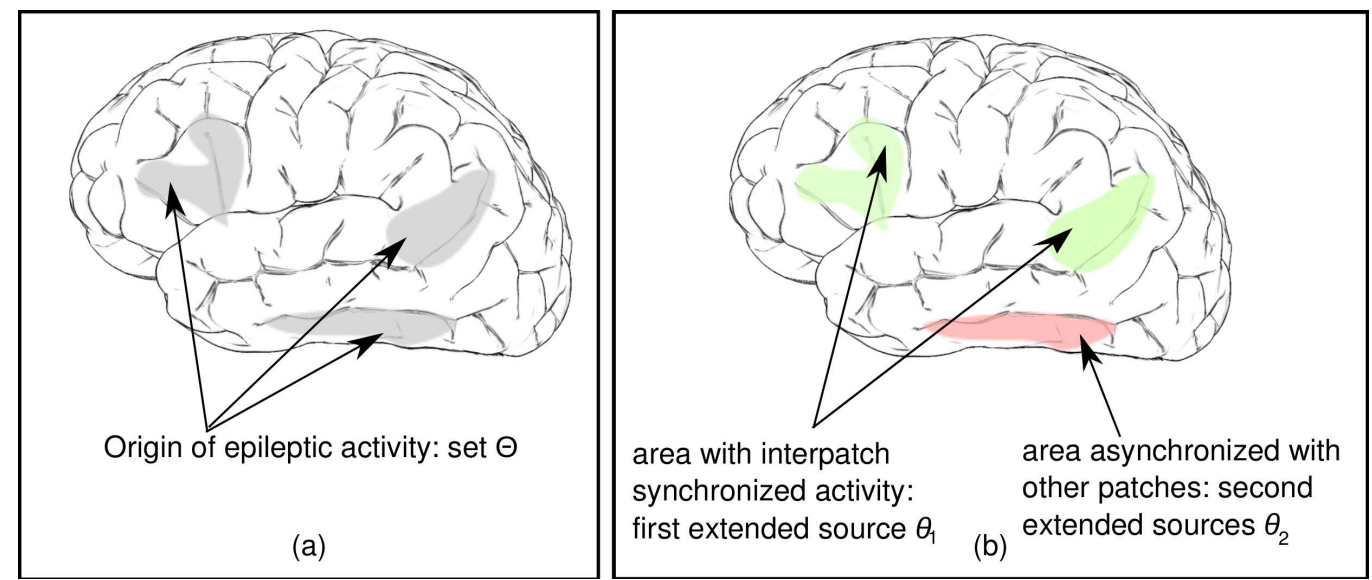

Figure 1: (a) Schematic representation of a cortex exhibiting a region of epileptic activity $\Theta$ (in gray). The entire cortex (including gray regions) generates also background activity. (b) An example of ExSo's configuration. On this schematic representation, the cortical area $\Theta$ exhibiting epileptic activity can be partitioned in two extended sources $\theta_{1}$ (in green) and $\theta_{2}$ (in red). In $\theta_{1}$, two spatially distinct patches are considered. The activity within and between the two patches are synchronized. Therefore $\theta_{1}$ will be considered as a single ExSo. $\theta_{2}$ is made of a single patch. The activity within this patch is synchronized but is independent from the activity in $\theta_{1}$. Therefore $\theta_{2}$ is considered as another ExSo. 
When the activity between two (or more) spatially distinct patches is desynchronized, then the method will regard them as two (or more) ExSos (see figure 1(b)). As the activity is highly synchronized within one ExSo, 2qExSo-MUSIC method makes the assumption that these activity is identical. Thus, the origin of epileptic activity $\Theta$ is partitioned into $P$ spatially distinct subsets $\theta_{p}$ such that all activity rising from $\theta_{p}$ is/are identical. The $P$ sets $\theta_{p}$ represent the $P$ extended sources and we have the property: for all $1 \leq p \leq P$, for all $\boldsymbol{\rho}$ in $\theta_{p},\left\{s_{\mathrm{e}}(\boldsymbol{\rho}, t)\right\}=\left\{s_{p}(t)\right\}$, where $\left\{s_{p}(t)\right\}$ is the activity associated with the $p$ th ExSo. Accordingly, in the EEG/MEG model (2), the epileptic activity can be factorized as

$$
\boldsymbol{x}(t)=\sum_{p=1}^{P} s_{p}(t) \sum_{\boldsymbol{\rho} \in \theta_{p}} \boldsymbol{a}(\boldsymbol{\rho})+\sum_{\boldsymbol{\rho} \in \Omega} \boldsymbol{a}(\boldsymbol{\rho}) s_{\mathrm{b}}(\boldsymbol{\rho}, t)+\boldsymbol{\nu}_{i}(t)
$$

The second term of this equation can be seen as a Gaussian noise of unknown spatial coherence. The resulting global noise $\boldsymbol{\nu}(t)=\sum_{\boldsymbol{\rho} \in \Omega} \boldsymbol{a}(\boldsymbol{\rho}) s_{\mathrm{b}}(\boldsymbol{\rho}, t) \boldsymbol{d} \boldsymbol{\rho}+$ $\boldsymbol{\nu}_{i}(t)$ is consequently a Gaussian noise of unknown spatial coherence and independent from the epileptic sources. In order to compact (3), we introduce the ExSo leadfield vector $\boldsymbol{h}(\theta)=\sum_{\boldsymbol{\rho} \in \theta} \boldsymbol{a}(\boldsymbol{\rho})$ that transcripts the contribution of an extended source $\theta$ with unitary activity to the scalp electrodes. An ExSo leadfield matrix $\boldsymbol{H}(\Theta)=\left[\boldsymbol{h}\left(\theta_{1}\right), \ldots, \boldsymbol{h}\left(\theta_{P}\right)\right]$ and an ExSo activity vector $\boldsymbol{s}(t)=\left[s_{1}(t), \ldots, s_{P}(t)\right]^{\top}$ are also introduced. Finally, equation (3) can be written

$$
\boldsymbol{x}(t)=\sum_{p=1}^{P} \boldsymbol{h}\left(\theta_{p}\right) s_{p}(t)+\boldsymbol{\nu}(t)=\boldsymbol{H}(\Theta) \boldsymbol{s}(t)+\boldsymbol{\nu}(t)
$$

\subsection{The 2q-ExSo-MUSIC method}

The $2 q$-ExSo-MUSIC method uses the $2 q$-th order $(q \geq 1)$ cumulants of the EEG/MEG data $\{\boldsymbol{x}(t)\}$. These cumulants are ordered in a $\left(N^{q} \times N^{q}\right)$ 
matrix $\boldsymbol{C}_{2 q, \boldsymbol{x}}$ also called the $2 q$-th order statistical matrix of the data (see Appendix A for technical details about the estimation of statistical matrices). In agreement with (4) and using the multilinearity property enjoyed by the cumulants, the $2 q$-th order statistical matrix of the data can be written as a function of $\Theta$ as (Chevalier et al., 2006)

$$
\boldsymbol{C}_{2 q, \boldsymbol{x}}=\boldsymbol{H}(\Theta)^{\otimes q} \boldsymbol{C}_{2 q, \boldsymbol{s}}\left[\boldsymbol{H}(\Theta)^{\otimes q}\right]^{\top}+\boldsymbol{C}_{2, \boldsymbol{\nu}} \delta[q-1]
$$

where $\boldsymbol{H}(\Theta)^{\otimes q}$ is the Kronecker product of the matrix $\boldsymbol{H}(\Theta)$ by itself $q-1$ times, $\boldsymbol{C}_{2 q, \boldsymbol{s}}$ is the $\left(P^{q} \times P^{q}\right) 2 q$-th order statistical matrix of $\{\boldsymbol{s}(t)\}$ and $\boldsymbol{C}_{2, \boldsymbol{\nu}}$ is the covariance matrix (second order statistical matrix) of the noise $\{\boldsymbol{\nu}(t)\}$. As HO statistics of Gaussian processes are zero, this covariance matrix is weighted by the Kronecker symbol $\delta[q-1]$. We define the signal subspace as $\operatorname{Span}\left\{\boldsymbol{H}(\Theta)^{\otimes q} \boldsymbol{C}_{2 q, \boldsymbol{s}}\left[\boldsymbol{H}(\Theta)^{\otimes q}\right]^{\top}\right\}$, which can be directly estimated from the data if $q>1$ since $\operatorname{Span}\left\{\boldsymbol{H}(\Theta)^{\otimes q} \boldsymbol{C}_{2 q, s}\left[\boldsymbol{H}(\Theta)^{\otimes q}\right]^{\top}\right\}=\operatorname{Span}\left\{\boldsymbol{C}_{2 q, \boldsymbol{x}}\right\}$, and from the data and the noise covariance if $q=1$ since $\operatorname{Span}\left\{\boldsymbol{H}(\Theta) \boldsymbol{C}_{2, s}[\boldsymbol{H}(\Theta)]^{\top}\right\}=$ $\operatorname{Span}\left\{\boldsymbol{C}_{2 \boldsymbol{x}}-\boldsymbol{C}_{2 \boldsymbol{\nu}}\right\}$.

To be able to identify the origin of epileptic activity, the $2 q$-ExSo-MUSIC method requires the following assumptions: i) the $P$ processes $\left\{s_{p}(t)\right\}$ are not Gaussian, ii) the dimension of the signal subspace has not reached its maximum (in the worst case, this surely holds if the number $P$ of extended sources is strictly inferior to the number $N$ of electrodes) and iii) the electrode setting has no ambiguities (meaning that each sensor brings its own information to the data). Under these hypotheses the vectors $\boldsymbol{h}(\theta)^{\otimes q}$, for which $\theta=\theta_{p}$, belong to the signal subspace. Moreover, for $q=1$, all vectors $\boldsymbol{h}(\theta)$ such that $\theta$ is a union of two or more sets $\theta_{p}$ also belong to the signal subspace. For $q>1$, the unions that enjoy this property depends on 
the correlation between the ExSo activity $\left\{s_{p}(t)\right\}$. But whatever $q$ and the correlation state of the sources, we are able to identify the origin of epileptic activity $\Theta=\bigcup_{1 \leq p \leq P} \theta_{p}$ by amalgamating all the candidate ExSo's $\theta$ such $\boldsymbol{h}(\theta)^{\otimes q}$ belongs to the signal subspace.

To check the belongness of $\boldsymbol{h}(\theta)^{\otimes q}$ to the signal subspace, a signal subspace projector $\boldsymbol{E}_{2 q, s} \boldsymbol{E}_{2 q, \boldsymbol{s}}^{\top}$ is built by EigenValue Decomposition (EVD) of $\boldsymbol{C}_{2 q, \boldsymbol{x}}-\boldsymbol{C}_{2, \boldsymbol{\nu}} \delta[q-1]$. More precisely, for $q>1, \boldsymbol{E}_{2 q, \boldsymbol{s}}$ is a matrix which column vectors are the eigenvector associated with the non-zero eigenvalues of $\boldsymbol{C}_{2 q} \boldsymbol{x}$. For $q=1$, the covariance matrix of the noise has to be known since $\boldsymbol{E}_{2, s}$ is then the matrix associated with the non-zero eigenvalues of $\boldsymbol{C}_{2, \boldsymbol{x}}-\boldsymbol{C}_{2, \boldsymbol{\nu}}$. Finally, in line with the MUSIC-like methods (Chevalier et al., 2006; Schmidt, 1986), the roots of the criterion $\Upsilon\left(\boldsymbol{E}_{2 q, \boldsymbol{s}}, \boldsymbol{h}(\theta)^{\otimes q}\right)=$ $1-\left(\left[\boldsymbol{h}(\theta)^{\otimes q}\right]^{\top} \boldsymbol{E}_{2 q, s} \boldsymbol{E}_{2 q, s^{\top}} \boldsymbol{h}(\theta)^{\otimes q}\right) /\left\|\boldsymbol{h}(\theta)^{\otimes q}\right\|_{2}^{2}$, also called metric, allow us to give an estimation, $\widehat{\Theta}$, of $\Theta$ :

$$
\widehat{\Theta}=\bigcup\left\{\theta, \Upsilon\left(\boldsymbol{h}(\theta)^{\otimes q}, \boldsymbol{E}_{2 q, \boldsymbol{s}}\right)=0\right\}
$$

However, in practice, the identification of $\widehat{\Theta}$ is difficult. Indeed, extended sources can theoretically be a free subset of potentially non-contiguous areas of the source space $\Omega$. Therefore, all the candidate sets $\theta$ representing all the possible topologies of extended sources cannot be exhaustively scanned and, as a correlate the comprehensive search of the roots of the metric $\Upsilon\left(\boldsymbol{h}(\theta)^{\otimes q}, \boldsymbol{E}_{2 q, \boldsymbol{s}}\right)$ cannot be achieved with a reasonable computational cost. In order to overcome this difficulty, we propose a low-cost optimization of the metric. This requires that the complex geometry of $\theta$ is parameterized with a relatively small number of parameters. An extended source of any shape can be approximated by the reunion of elementary simple-shaped 
subsets. Subsets with circular shapes are good candidates since they can be entirely described by only two parameters, say the center and the radius. We propose a non-trivial identification of these subsets based on the thresholding of the metric $\Upsilon\left(\boldsymbol{E}_{2 q, \boldsymbol{s}}, \boldsymbol{h}(\tilde{\theta})^{\otimes q}\right)$ where $\tilde{\theta}$ is a circular-shaped candidate subset. More precisely, the candidate subsets $\tilde{\theta}$ for which the metric is lower than a given threshold $\lambda$ are united in order to estimate simultaneously all the actual extended sources. A schematic representation of this concept is given in figure $2(\mathrm{a})$. In this situation, the resulting $2 q$-ExSo-MUSIC becomes

$$
\widehat{\Theta}^{2 q-\mathrm{ExSo-MUSIC}}(\lambda)=\bigcup\left\{\tilde{\theta}, \Upsilon\left(\boldsymbol{h}(\tilde{\theta})^{\otimes q}, \boldsymbol{E}_{2 q, \boldsymbol{s}}\right) \leq \lambda\right\}
$$

The validity of such a result is provided in a probabilistic sense in Appendix B. The estimated set of extended sources $\widehat{\Theta}^{2 q \text {-ExSo-MUSIC }}(\lambda)$ depends on the threshold $\lambda$. This parameter can be adjusted for a good compromise between the specificity and sensitivity of the estimator. As shown in figure 2(b), for low values of $\lambda$ the estimator has a low sensitivity but a high specificity. This means that the estimated set of extended sources is included in the actual set $\Theta$ (true positive) and does not encompass it (false positive). When $\lambda$ increases, the sensitivity increases while the specificity decreases.

\subsection{Simulation protocol}

The $2 q$-ExSo-MUSIC method $(q=1,2)$ was evaluated on 31 channels EEG data generated from a model developed in our team (Cosandier-Rimélé et al., 2007, 2008; Wendling et al., 2000), which main features are briefly summarized below. In order to construct a source space for our simulations, we built a mesh of the cortical surface from a 3D MRI T1 image of a patient (BrainVisa, SHFJ, Orsay, France). This mesh was composed of 

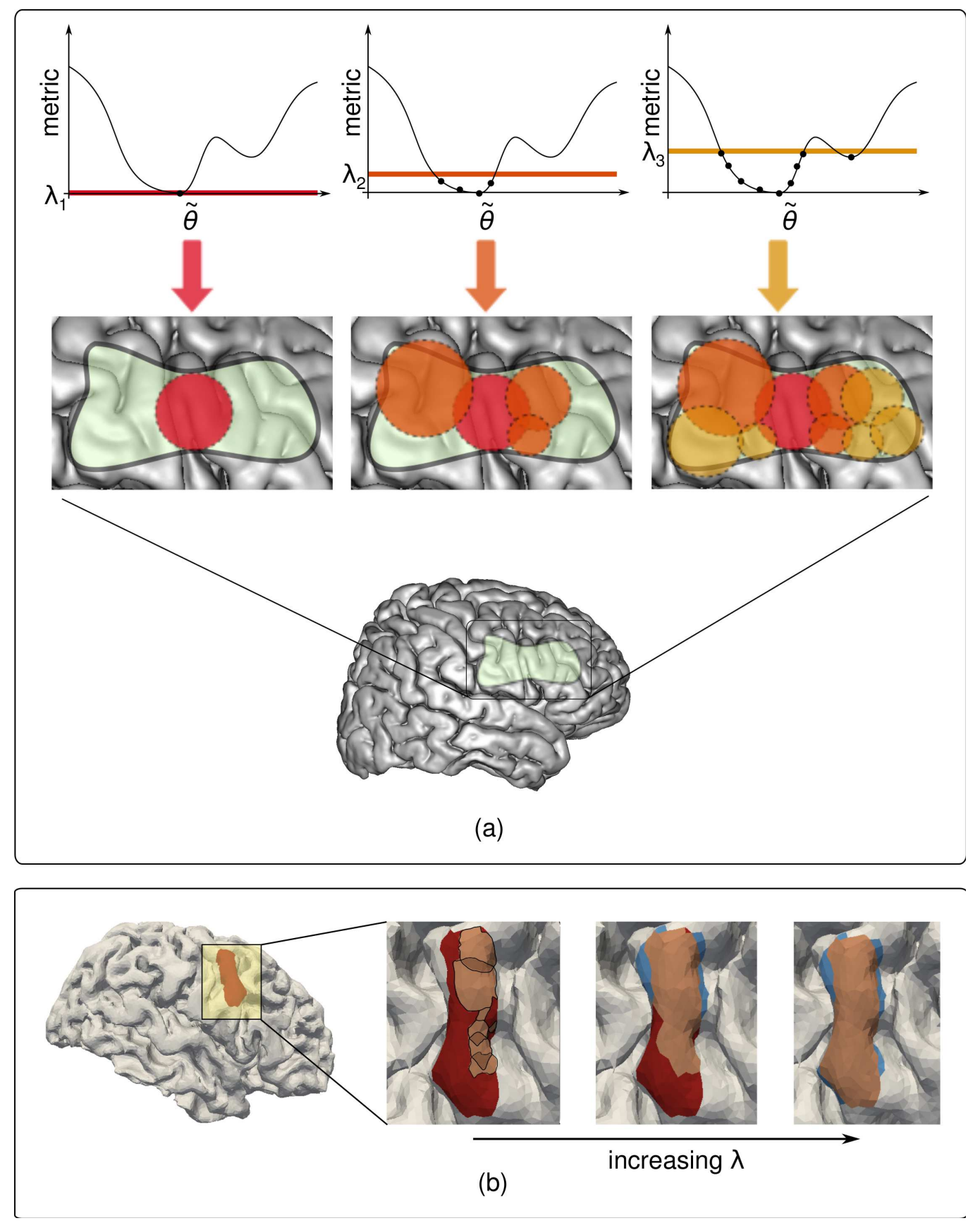

Figure 2: The ExSo-MUSIC principle. (a) Schematic representation of the ExSo-MUSIC principle. When the threshold $\lambda$ increases, the number of concatenated circular sets $\tilde{\theta}$ increases, enlarging the surface of the estimated extended source. (b) Example of result using 4-ExSo-MUSIC method. The actual extended source $\Theta$ is red. The true estimated parts $\hat{\Theta}^{4-\operatorname{ExSo}-\operatorname{MUSIC}}(\lambda) \cap \Theta$ are beige, and the false estimated parts $\hat{\Theta}^{4-E x S o-M U S I C}(\lambda)-\hat{\Theta}^{4-E x S o-M U S I C}(\lambda) \cap \Theta$ are bluel $2 \mathrm{n}$ practice, as the source space is a triangular mesh of the cortical surface, we cannot construct perfectly circular disks. Instead we consider pseudo-disks, made of one or several triangles and parameterized by the disk area and the position of its center, as defined by the position of the germ triangle. For a mesh with sufficiently high resolution, any ExSo can be approximated by the union of these pseudo-disks. 
$M=143022$ triangles of mean surface $1 \mathrm{~mm}^{2}$. A current dipole was set up at the barycenter $\boldsymbol{\rho}_{m}$ of each triangle and oriented orthogonally to the triangle surface, leading to a field of current dipoles. Our simulation source space was defined by $\left\{\boldsymbol{\rho}_{m}, 1 \leq m \leq M\right\}$ and corresponded to a cortical mesh $\Omega$ with a resolution of about $1 \mathrm{~mm}^{2}$.

Time activities $\left\{s\left(\boldsymbol{\rho}_{m}, t\right), 1 \leq m \leq M\right\}$ of all dipoles of the source space were generated with a realistic neuronal population model (Wendling et al., 2000 ), in which excitation and inhibition parameters of each population can be adjusted to obtain either interictal epileptic spikes or background activity. With an appropriate setting of coupling parameters bewteen populations, we built a set of highly-correlated interictal spike activities. As illustrated in figure 3(a), these activities were assigned to a set of triangle (actual ExSo) manually outlined with a mesh vizualisation software (Paraview, Kitware Inc., NY, US). Uncorrelated background activities were attributed to the other triangles (figure 3(a)). According to the scenario considered, we varied the size, location, number or configuration of the source(s). From this setup, we built a spatio-temporal source matrix $\boldsymbol{S}$ where the $m$ th line stands for the activity of the $m$ th dipole for $K=9984$ temporal samples (39 s with sampling frequency of $256 \mathrm{~Hz})$.

In order to generate the simulated EEG data, we used a realistic head model, that consists in three nested homogenous volume realistically shaping the brain, the skull and the scalp (figure 3(b)). The conductivity of each medium was fixed to $0.33 \mathrm{~S} / \mathrm{m}, 0.0082 \mathrm{~S} / \mathrm{m}$ and $0.33 \mathrm{~S} / \mathrm{m}$, respectively (Gonçalves et al., 2003). The inter-medium surfaces were extracted from the segmentation of a T1-weighted 3D-MRI and meshed by 2440 triangles each 
(a)

Cortical spike-like activities within the ExSo
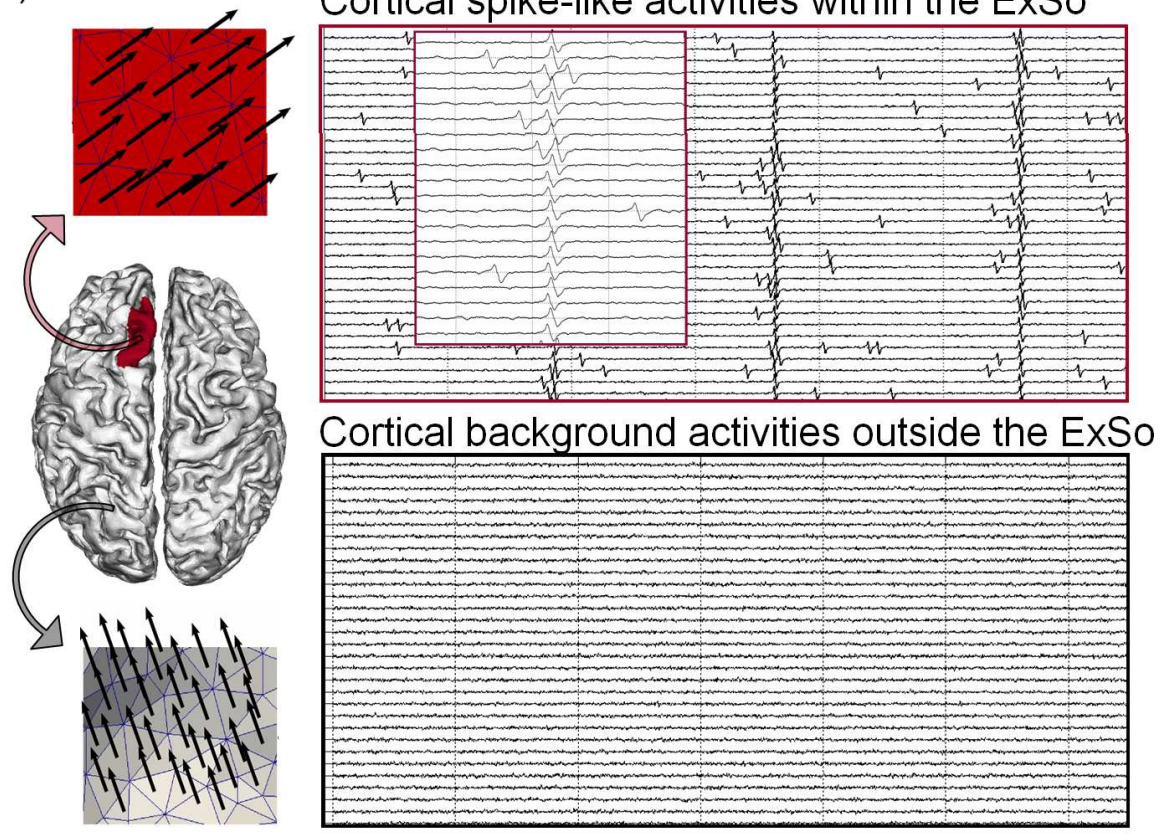

(b)

\section{Simulated scalp EEG}
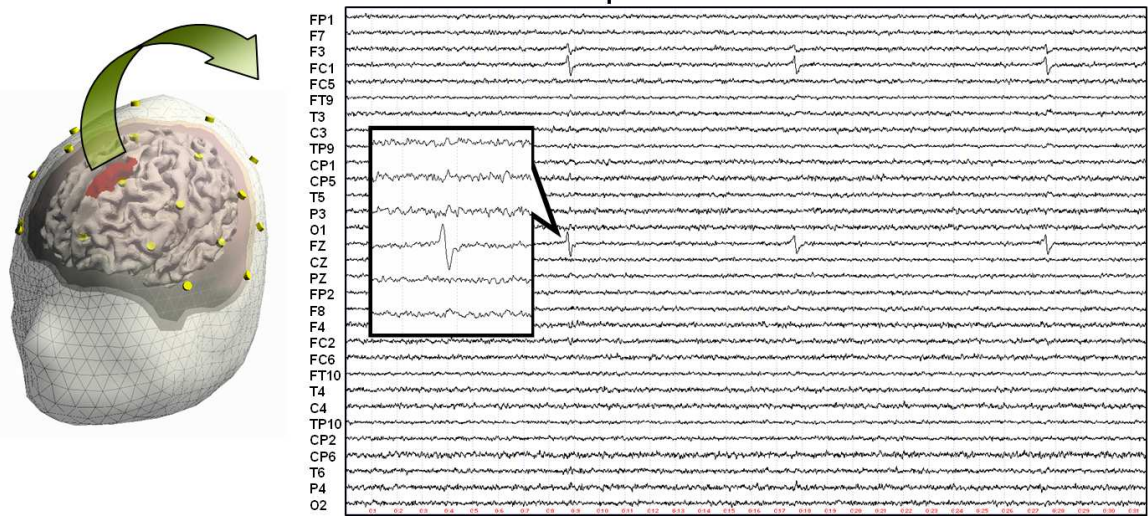

Figure 3: Simulation of scalp EEG spikes. (a) An extended source of 1000 triangles $\left(\approx 10 \mathrm{~cm}^{2}\right)$ was manually defined on a mesh of the cortical surface using Paraview (Paraview, Kitware Inc., NY, US). Dipoles are placed all over the mesh at the barycenter of each triangle and oriented orthogonal to the triangle surface. Within the source, each dipole is assigned with a spike-like activity. For the whole source, a series of 1000 highly synchronized activities is generated using the neuronal population model (50 are displayed here). In the remaining part of the mesh dipoles are assigned with uncorrelated background activity. (b) Using this source configuration, a realistic head model and a given set of 31 electrodes, EEG data were computed solving numerically the forward problem. In this particular case, epileptic spikes generated from the left superior frontal ExSo are clearly identified on F3, FC1 and Fz. 
(ASA, ANT, Enschede, Netherlands). From this head model, the forward problem was then numerically calculated for each triangle (ASA, ANT, Enschede, Netherlands), to obtain the leadfield matrix $\boldsymbol{A}=\left[\Delta_{1} \boldsymbol{a}\left(\boldsymbol{\rho}_{1}\right), \ldots, \Delta_{m} \boldsymbol{a}\left(\boldsymbol{\rho}_{M}\right)\right]$ where $\boldsymbol{a}\left(\boldsymbol{\rho}_{m}\right)$ is the leadfield vector associated with the $m$-th triangle and $\Delta_{m}$ the triangle surface. The $\boldsymbol{A}$ matrix gives the contribution of each dipole of the mesh at the level of 31 scalp electrode position (19-20 standard 10-20 electrodes plus additional electrodes at FC1, FC2, FC5, FC6, CP1, CP2, CP5, FT9, FT10, P9 and P10). The spatio-temporel matrix of simulated EEG/MEG data is given by $\boldsymbol{X}=\boldsymbol{A} \boldsymbol{S}+\boldsymbol{V}$, where $\boldsymbol{V}$ is a zero-mean Gaussian spatio-temporal matrix of the instrumental noise $\boldsymbol{\nu}_{i}(t)$.

As previously reported (Grova et al., 2006), we used ROC curves (Metz, 1986) to measure the performance of the $2 q$-ExSo-MUSIC method $(q=1,2)$. This criterion represents a graph of the mathematical expectation of the True Positive Fraction, $E[\operatorname{TPF}(\lambda)]$, as a function of the mathematical expectation of the False Positive Fraction, E[FPF $(\lambda)]$. Estimations, $\overline{\mathrm{TPF}}$ and $\overline{\mathrm{FPF}}$, of these mathematical expections are computed by averaging the results of 50 monte-carlo realizations. For the $\ell$-th realization, $\operatorname{TPF}_{\ell}(\lambda)$ and $\operatorname{FPF}_{\ell}(\lambda)$ were expressed as

$$
\begin{aligned}
\operatorname{TPF}_{\ell}(\lambda) & =\frac{\operatorname{surf}\left\{\hat{\Theta}_{\ell}(\lambda) \cap \Theta\right\}}{\operatorname{surf}\{\Theta\}} \\
\operatorname{FPF}_{\ell}(\lambda) & =\frac{\operatorname{surf}\left\{\hat{\Theta}_{\ell}(\lambda)\right\}-\operatorname{surf}\left\{\hat{\Theta}_{\ell}(\lambda) \cap \Theta\right\}}{\operatorname{surf}\{\Omega\}-\operatorname{surf}\{\Theta\}}
\end{aligned}
$$

where surf $\{$.$\} is the area of a given cortical region and \hat{\Theta}_{\ell}(\lambda)$ is the estimated set of extended sources at realization $\ell$. In order to display the results in a compact way, only the normalized Area under the ROC Curve $(\mathrm{AuC})$, for $\overline{\mathrm{FPF}}$ from 0 to 0.1 , is represented. AuC values range between 0 (the worst 
result) and 1 (the best result).

In order to analyze the performance of the methods as a function of the spike amplitude on the EEG data, the Mean Spike-to-Background Ratio (MSBR) is introduced as

$$
\operatorname{MSBR}=10 \log \mathrm{E}\left[\left\|\boldsymbol{x}_{s}(t)\right\|^{2}\right] / \mathrm{E}\left[\left\|\boldsymbol{x}_{b}(t)\right\|^{2}\right]
$$

where $\left\{\boldsymbol{x}_{s}(t)\right\}$ stands for an EEG epoch containing a spike (negative peak from baseline to baseline) and $\left\{\boldsymbol{x}_{b}(t)\right\}$ is an EEG epoch containing only background. In the EEG generation, the MSBR was tuned by setting the amplitudes of the spikes at the level of the sources $\boldsymbol{S}$.

\section{Results}

\subsection{Influence of $M S B R$}

In order to study the performance of the methods as a function of the MSBR in simulated EEG data, an ExSo made of a single patch of $1000 \mathrm{~mm}^{2}$ was placed in the left occipital gyrus. EEG epochs for each considered MSBR are displayed in figure 4(a). The performance of the methods is reported in figure 4(b). For the highest value of MSBR (18.3 dB) 2-ExSo-MUSIC and 4-ExSo-MUSIC showed the best performance. As the MSBR decreased, the AuC of 4-ExSo-MUSIC stayed stable . On the contrary, the performance of the 2-ExSo-MUSIC method decreased quickly from a MSBR equal to $10.7 \mathrm{~dB}$ and reached zero for $3.3 \mathrm{~dB}$. The AuC of 2-MUSIC and 4-MUSIC followed 2ExSo-MUSIC and 4-ExSo-MUSIC ones, respectively, but with an important shift to the bottom. This result showed i) the better robustness of $\mathrm{HO}$ 
methods with respect to the MSBR and ii) the better accuracy of the $2 q$ ExSo-MUSIC algorithm with respect to the 2q-MUSIC approach, for a given value of $q$.

\subsection{Influence of the ExSo area}

In order to study the performance of the methods as a function of the area of the source, an ExSo made of a single patch was placed in the left dorsolateral frontal cortex. The size of this patch varied from 50 to 2000 $\mathrm{mm}^{2}$ (examples are illustrated on fig. 5(a)). The AuC for all four methods are displayed on figure 5(b) along with with the corresponding MSBR values (as the patch area has a direct influence on the MSBR in simulated EEGs). For all the ExSo areas, the performance of 4-ExSo-MUSIC was always higher than that of other methods. However, ExSo's with a size less than $400 \mathrm{~mm}^{2}$ $(\mathrm{MSBR}<2 \mathrm{~dB})$ remained poorly estimated by 4-ExSo-MUSIC $(\mathrm{AuC}<0.3)$ and by 4 -MUSIC $(\mathrm{AuC}<0.1)$. SO methods did not retrieve any of the ExSo's when their area was inferior to $800 \mathrm{~mm}^{2}$ (MSBR $<4 \mathrm{~dB}$ ) while 2-MUSIC, 4-MUSIC and 2-ExSo-MUSIC displayed a stable efficiency for ExSo areas ranging from 800 to $1200 \mathrm{~mm}^{2}$. The ability of 4-ExSo-MUSIC to estimate the actual ExSo was considerably increased up to $\mathrm{AuC}$ values around 0.95. 4-MUSIC was more efficient than SO algorithms in all instances except for ExSo's over $1750 \mathrm{~mm}^{2}$ for which 2-ExSo-MUSIC slightly exceeded 4-MUSIC. FInally, 2-MUSIC always showed the poorest performance.

\subsection{Influence of the ExSo location}

In order to study the influence of the ExSo location on the efficiency of the four algorithms, ExSo's made of single patch were placed successively 


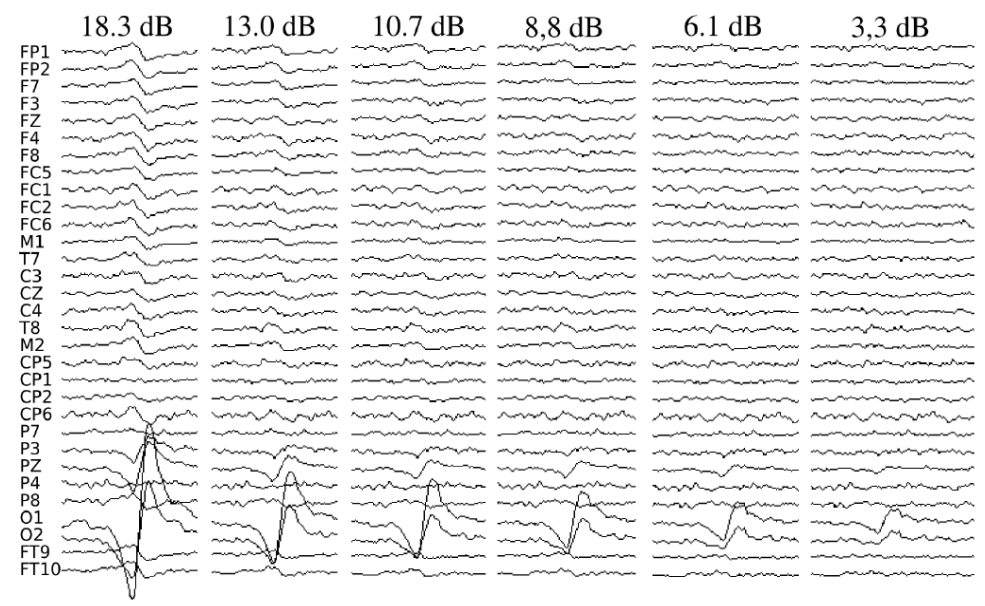

(a)

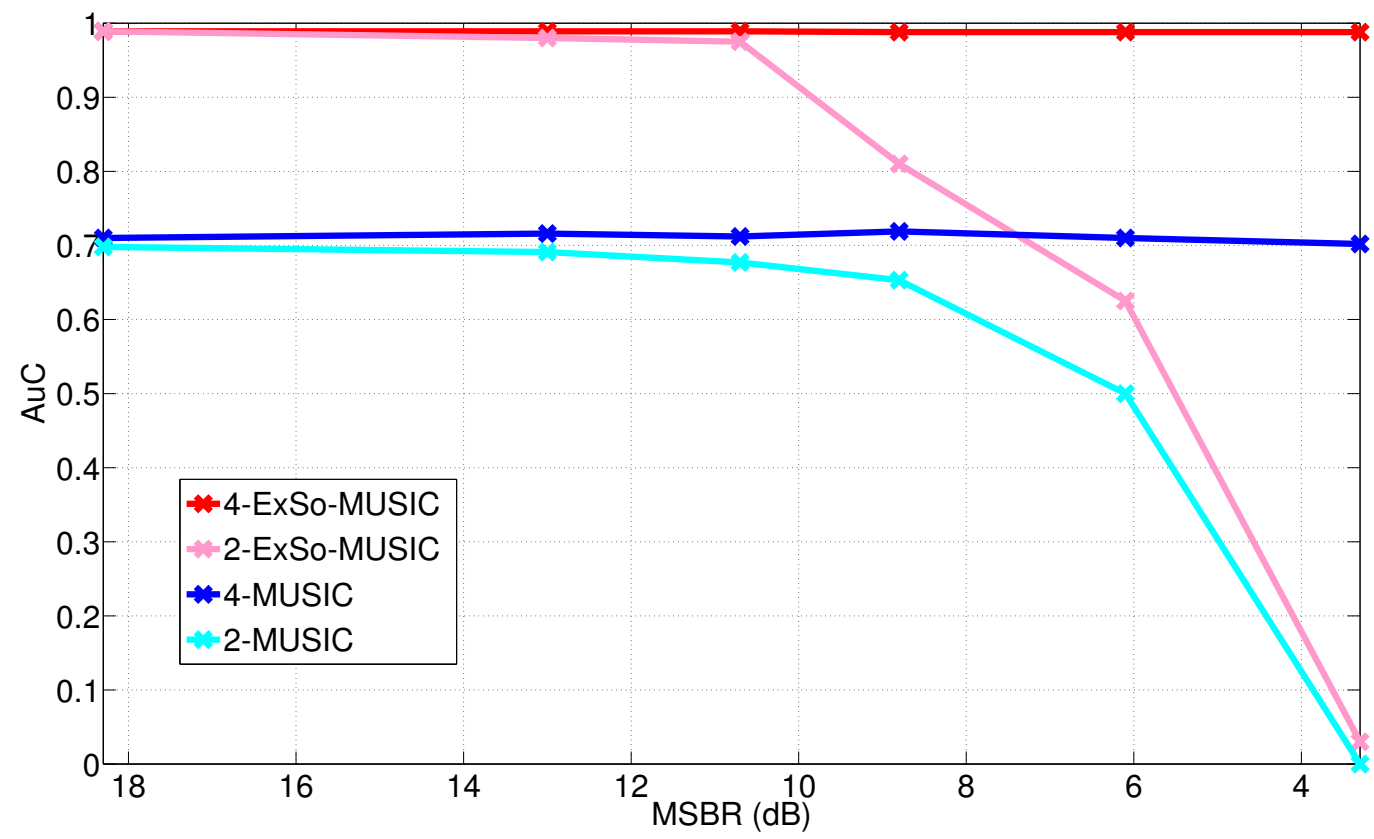

(b)

Figure 4: Performance of the source localization approaches with respect to the MSBR. (a) In this study, data were simulated from one "single-patch" ExSo of $1000 \mathrm{~mm}^{2}$ placed in the left lateral occipital region. Six different values of MSBR (from 18.3 to $3.3 \mathrm{~dB}$ ) are illustrated. (b) For the $2 q$-ExSo-MUSIC and $2 q$-MUSIC $(q=1,2)$ methods, the Area under the ROC Curve $(\mathrm{AuC})$ is plotted as a function of the MSBR. 


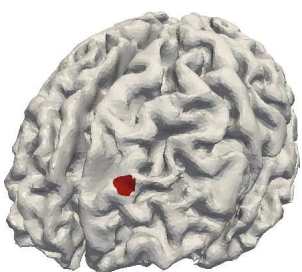

$50 \mathrm{~mm}^{2}$

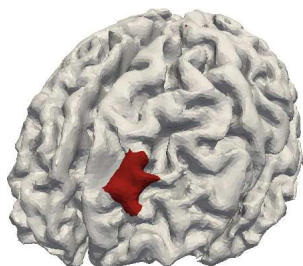

$200 \mathrm{~mm}^{2}$

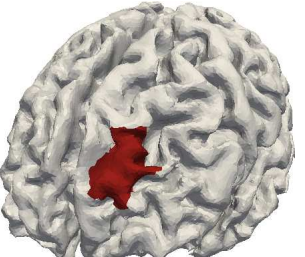

$1000 \mathrm{~mm}^{2}$

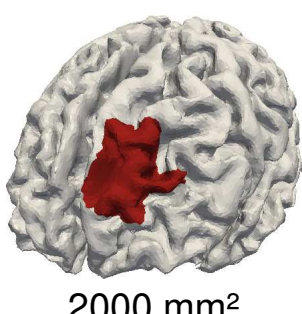

$2000 \mathrm{~mm}^{2}$

(a)

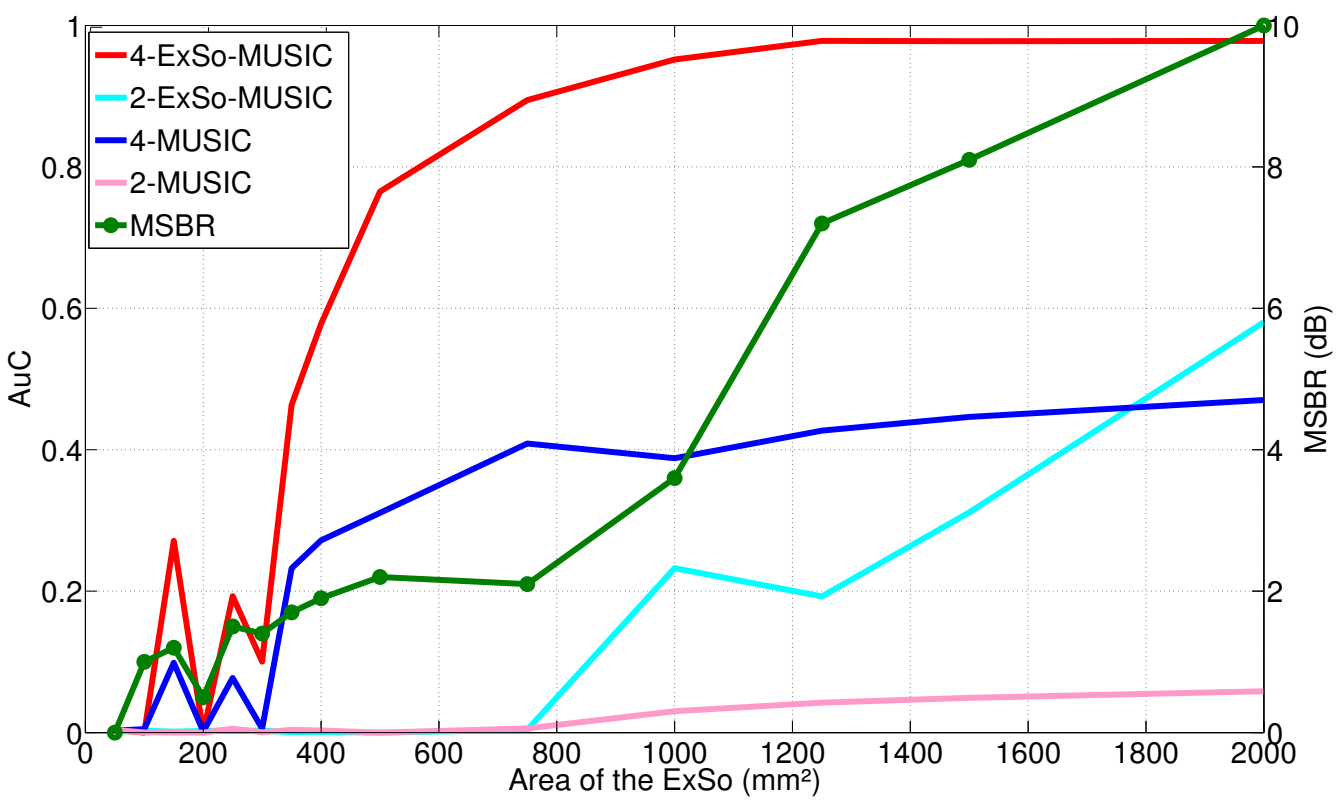

(b)

Figure 5: Performance of the source localization approaches with respect to the area of the ExSo. (a) In this study, data were simulated from one "single-patch" ExSo located in the left superior frontal region. The source area varied from 50 to $2000 \mathrm{~mm}^{2}$. Four different areas $\left(50,200,1000\right.$ and $2000 \mathrm{~mm}^{2}$ ) are illustrated. (b) AuC of the 2q-ExSo-MUSIC and 2q-MUSIC $(q=1,2)$ methods as a fonction of the ExSo area. The MSBR is also reported as it is relied to the ExSo area. 
in nine different brain areas of the left hemisphere (figure 6(a)). Lateral localizations (frontodorsal, occipital, precentral, middle temporal gyri and temporal pole) as well as mesial ones (cingulate, orbitofrontal, basal temporal gyri and parahippocampal gyrus) were considered. This study was repeated three times for an ExSo area of 315, 500 and $1000 \mathrm{~mm}^{2}$.

Values of $\mathrm{AuC}$ for all ExSo locations are reported on figure 6(b) along with MSBR. For all ExSo's but the one of $315 \mathrm{~mm}^{2}$ located in cingulate gyrus, the 4-ExSo-MUSIC algorithm showed the best performance and HO methods remains largely superior to the others ones. For $1000 \mathrm{~mm}^{2}$ ExSo's, the superiority of 4-ExSo-MUSIC over the other algorithms was particularly marked for ExSo's located in deep brain regions (orbitofrontal, temporobasal gyri as well as hippocampus) or regions located close to the midline (frontodorsal gyrus). For more lateral ExSo's (occipital, temporal or precentral gyri) with small as well as larger sizes, 4-ExSO-MUSIC and 4-MUSIC showed closer performance. Finally, ExSo's of 315 and 500 located in hippocampus and orbitofrontal gyrus were not localized by any of the algorithms, or poorly retrieved $(\mathrm{AuC}<0.2)$ by 4 -ExSo-MUSIC. 

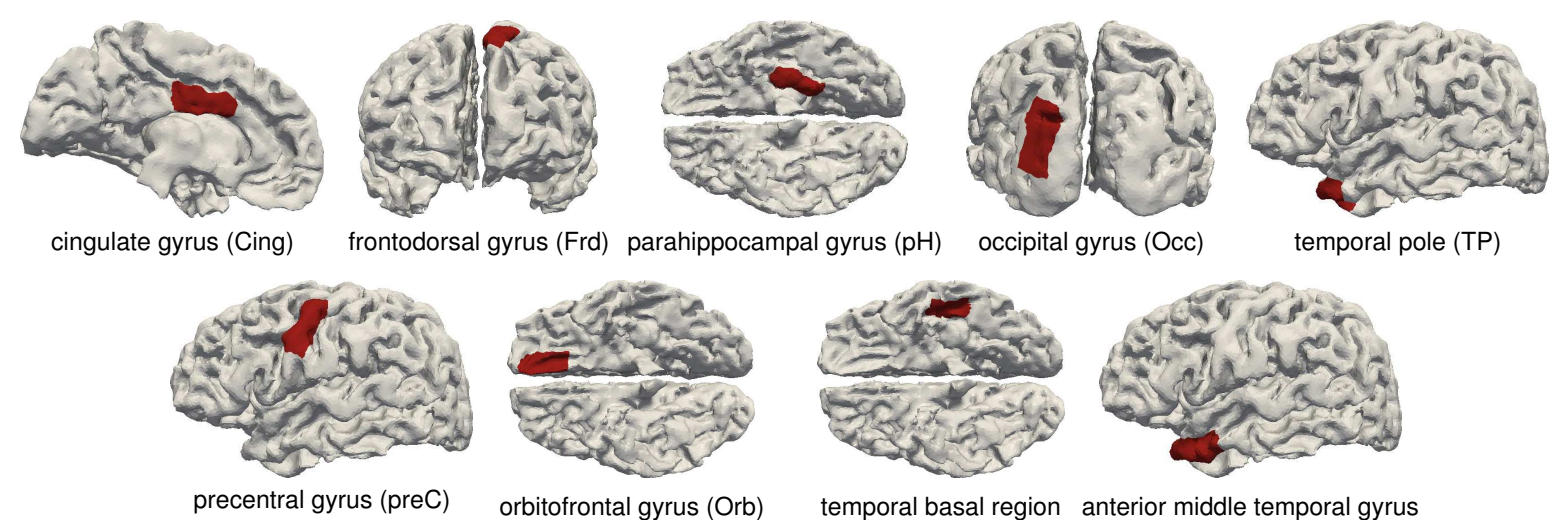

temporal basal region anterior middle temporal gyrus

(Tbas)

(aMT)

(a)

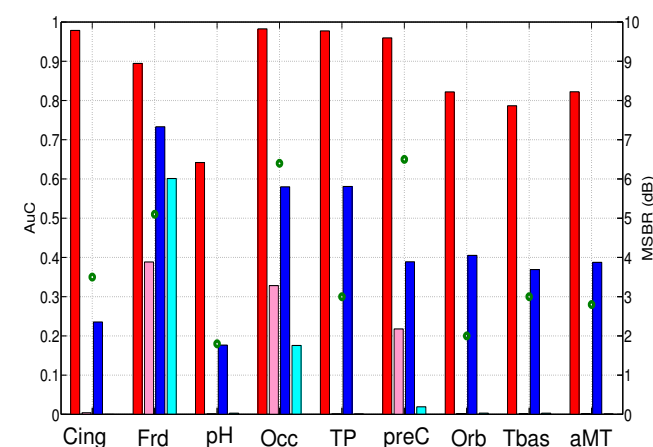

$1000 \mathrm{~mm}^{2}$

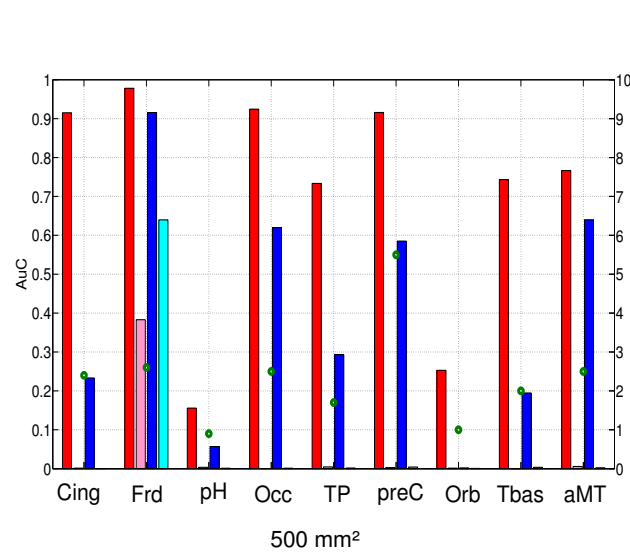

(b)

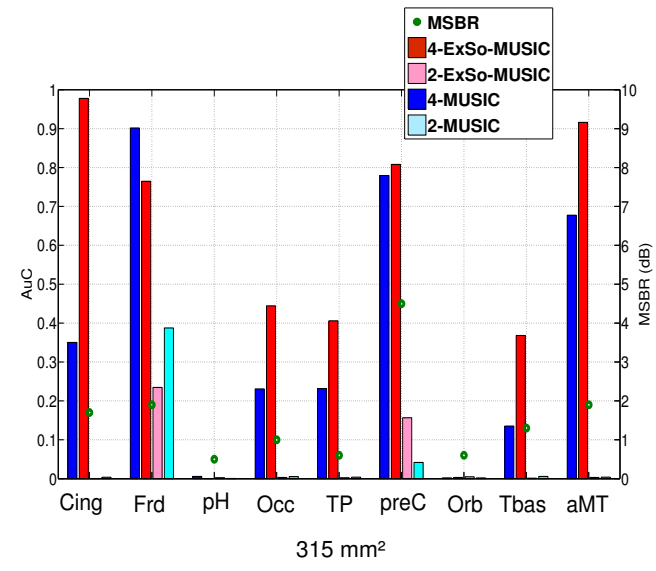

Figure 6: Performance of the source localization approaches with respect to the location of the ExSo. (a) In this study nine different source locations and three different areas $\left(315,500\right.$ and $\left.1000 \mathrm{~mm}^{2}\right)$ were used to simulate the data. The nine source locations are illustrated for an ExSo of $500 \mathrm{~mm}^{2}$. (b) AuC of the $2 q$-ExSo-MUSIC and $2 q$-MUSIC $(q=1,2)$ methods as a fonction of the ExSo location and for ExSo area of 315,500 and $1000 \mathrm{~mm}^{2}$. 
Illustrative examples of the localization results are given in figure 7 for a fixed $\overline{\text { FPF }}$ value of $5 \cdot 10^{-3}$. This shows that the localization accuracy of the 4-ExSo-MUSIC method was highly superior to that of the other approaches. Interestingly, with 4-ExSo-MUSIC, mislocated dipoles were contiguous with the external edge of the actual ExSo, while with classical MUSIC-like methods, mislocated dipoles were more scattered, some of them being not contiguous with the actual source.

\subsection{Influence of the number of ExSos and of their configuration}

For this study, we have considered three different ExSo configurations: i) two spatially distinct patches, located in left inferior and superior temporal gyri, with non-synchronized dipole activities between the two patches (i.e. two "single-patch" ExSos, see figure 8(a)), ii) the two same patches but with synchronized dipole activities between patches (i.e. one "double-patch" ExSo, see figure $8(\mathrm{~b})$ ), and iii) the two same patches with synchronized dipole activities between patches and an additional patch, located in the inferior frontal gyrus, which dipole activities are independent from those of the two other patches (i.e. one "double-patch" ExSo and one "single-patch" ExSo, see figure $8(\mathrm{c}))$.

Table 1 and figure 8 provide the $\mathrm{AuC}$ values and illustrate the localization accuracy of the four approaches, respectively. For all three source configurations considered, the 4-ExSo-MUSIC approach was able to retrieve accurately the actual patches, whether interpatch activities have been set synchronous or not. As for the previous study, mislocated dipoles were contiguous with the external edge of the actual ExSos. A smaller area of the actual ExSos was retrieved with the 4-MUSIC approach. This method also mislocated dipoles 

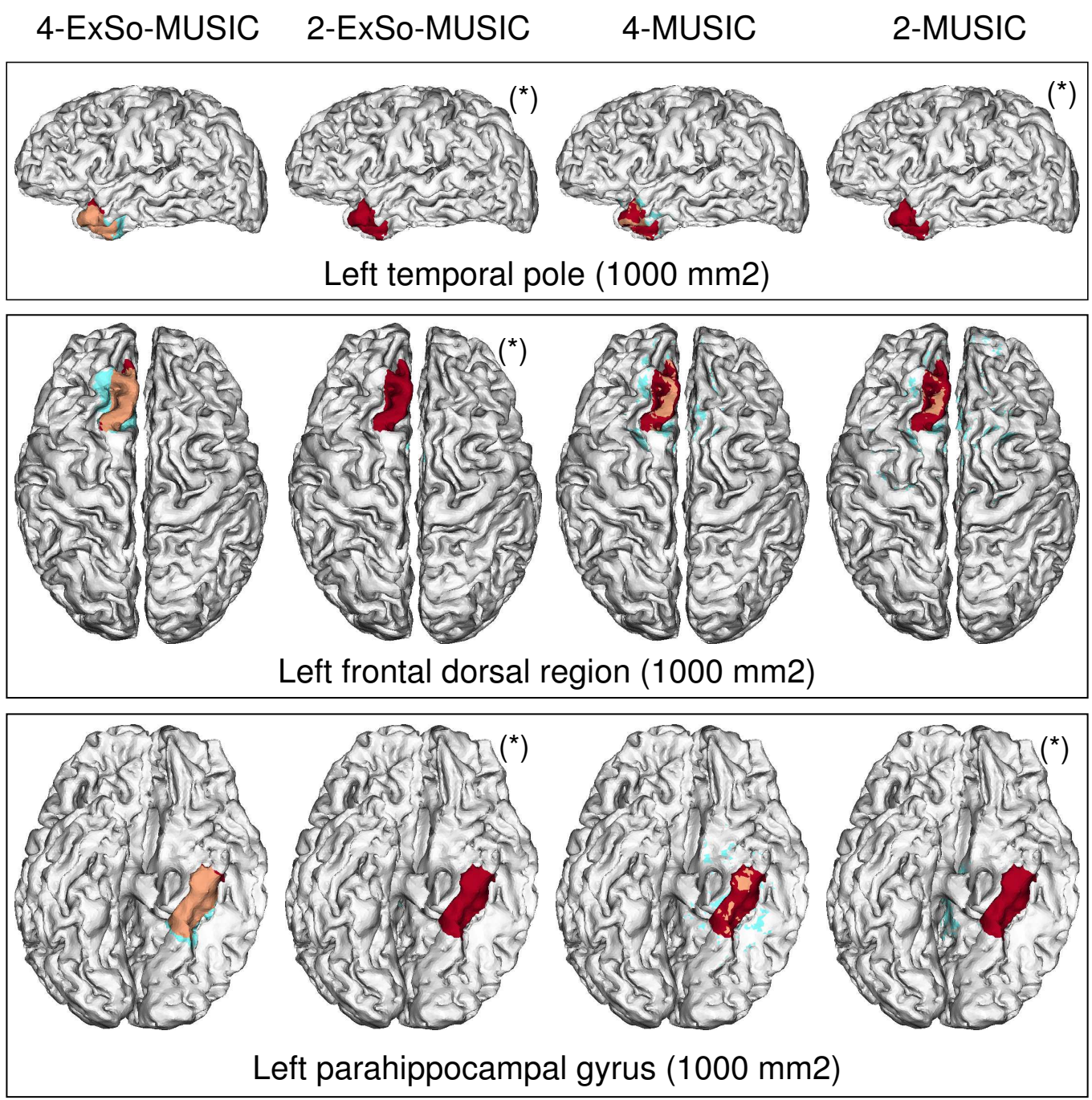

real ExSo

Well-estimated parts

(i.e. inside the ExSo)

Misestimated parts

(i.e. outside the ExSo)

Figure 7: Examples of localization results of 4-ExSo-MUSIC, 2-ExSo-MUSIC, 4-MUSIC and 2-MUSIC. The threshold was choosen such that $\overline{\mathrm{FPF}} \approx 5 \cdot 10^{-3}$ (this corresponds to an area of about $350 \mathrm{~mm}^{2}$ ). (a) $1000 \mathrm{~mm}^{2}$ ExSo in the left pole. (b) $1000 \mathrm{~mm}^{2}$ ExSo in the left frontodorsal gyrus. (c) $1000 \mathrm{~mm}^{2}$ ExSo in the left parahippocampus gyrus. $\left(^{*}\right)$ Misestimated parts are located in the cingulate region. 


\begin{tabular}{|l|c|c|c|c|}
\hline & 4-ExSo-MUSIC & 2-ExSo-MUSIC & 4-MUSIC & 2-MUSIC \\
\hline $\begin{array}{l}\text { (a) two "single-patch" ExSo's of } \\
1000 \mathrm{~mm}^{2} \text { each, located in the } \\
\text { superior and anterior temporal gyri }\end{array}$ & 0.987 & 0.042 & 0.534 & 0 \\
\hline $\begin{array}{l}\text { (b) one "double-patch" ExSo (two } \\
\text { patches of } 1000 \mathrm{~mm}^{2} \text { with } \\
\text { synchronized activity located as in (a)) }\end{array}$ & 0.961 & 0.033 & 0.476 & 0 \\
\hline $\begin{array}{l}\text { (c) one "double-patch" ExSo (same as in } \\
\text { (b)) and one "single-patch" ExSo of 1000 } \\
\text { mm² located in the inferior frontal gyrus }\end{array}$ & 0.953 & 0.026 & 0.372 & 0 \\
\hline
\end{tabular}

Table 1: AuC for all four localization methods obtained in the context of three multipatch ExSo configurations.

in a more scattered area. SO approaches displayed quasi-null performance (2-ExSo-MUSIC approach) or failed (2-MUSIC). 


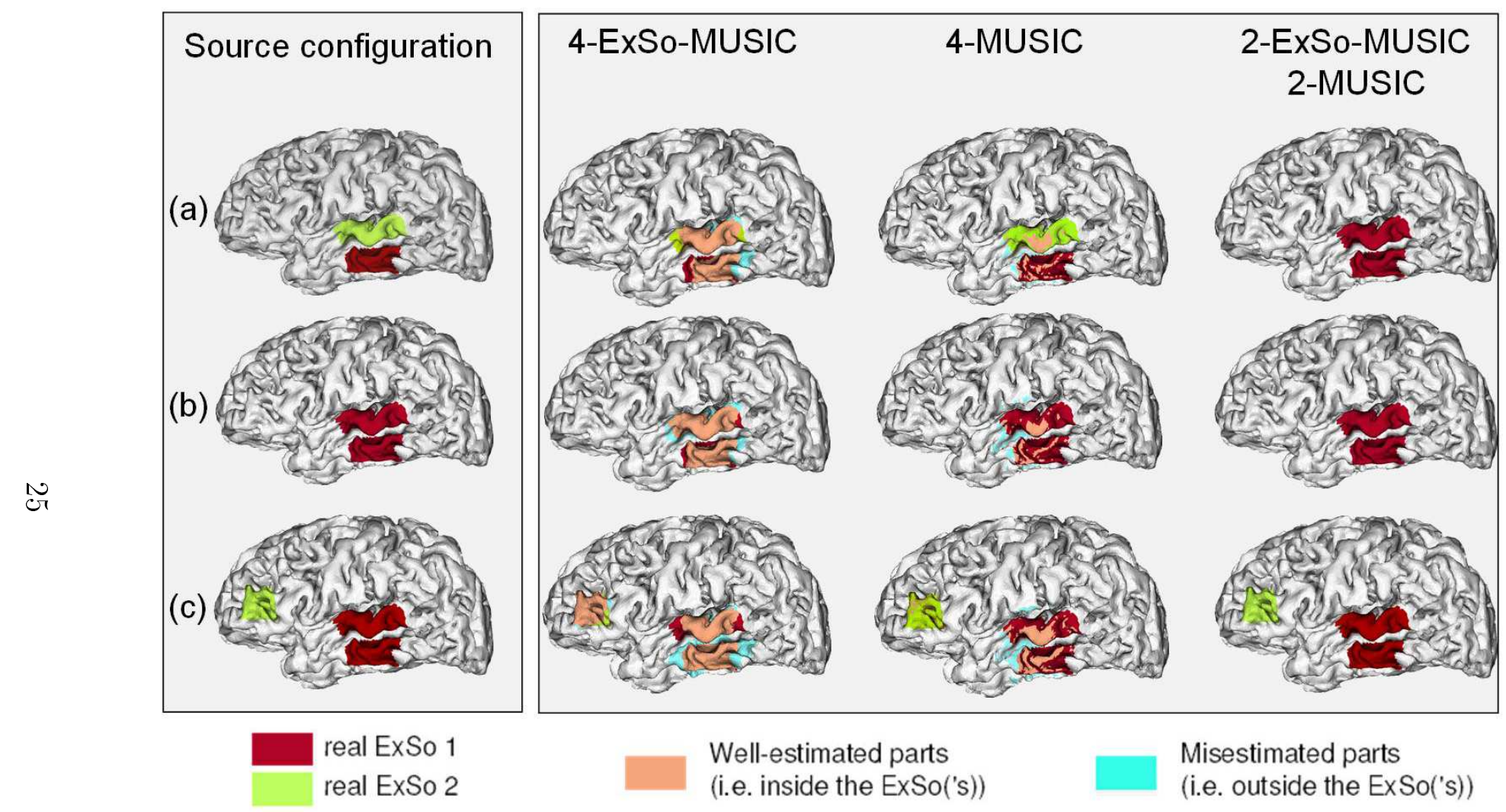

Figure 8: Localization results of the $2 q$-ExSo-MUSIC and $2 q$-MUSIC $(q=1,2)$ methods in the context of three multi-patch configurations. (a) two "single-patch" ExSo's (i.e. with interpatch independent activity) located in the superior and middle temporal gyrus. (b) one "double-patch" ExSo (i.e. with interpatch synchronized activity). (c) one "double-patch" ExSo (i.e. with interpatch synchronized activity) and one "single-patch" ExSo (i.e. withe activity independent from the first two) located in the middle frontal gyrus. 


\subsection{Influence of modeling errors}

In practice, some errors may occur in the forward model. The HO MUSIC-like methods have been shown to be more robust with respect to modeling errors than SO-MUSIC-like methods in particular in a multi-sources context (Birot et al., 2010; Chevalier et al., 2006, 2007). We studied the influence modeling error by displacing scalp electrodes from their original 10-20 position $(9(\mathrm{a}))$. For each electrode position error $(0.00 \pm 0.00,2.02 \pm 0.65$, $6.92 \pm 2.60$ and $11.58 \pm 5.52 \mathrm{~mm})$ EEG signals were simulated from two "single-patch" ExSos of $1000 \mathrm{~mm}^{2}$ located in the left anterior frontal and inferior parietal region. For this study inter-patch dipole activities were independent. The MSBR was fixed to a high value $(15 \mathrm{~dB})$ in order to not disadvantage the SO method with respect to the HO methods. Thus only the influence of modeling errors is involved. The localization results, expressed as a function of the mean electrode position error, are given in figure 9 (b). The performance of the FO methods stayed almost stable until an electrode position errors reached $7 \mathrm{~mm}$. On the contrary, $\mathrm{AuC}$ values of $\mathrm{SO}$ methods significantly decreased as soon as errors (even of small amplitude) in the electrode position occurred. For a given statistical order, the 2q-ExSo-MUSIC algorithm remained more accurate than the $2 q$-MUSIC method for all values of electrode position error.

\section{Discussion and Conclusion}

The proposed 2q-ExSo-MUSIC method allows for the localization of spatially extended sources from non-invasive electromagnetic recordings, by combining both the use of a novel principle (ExSo-MUSIC) and that of HO 


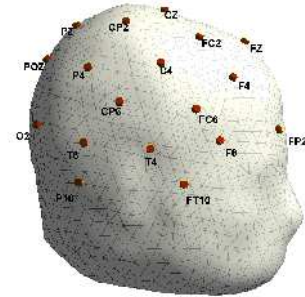

Initial electrode position
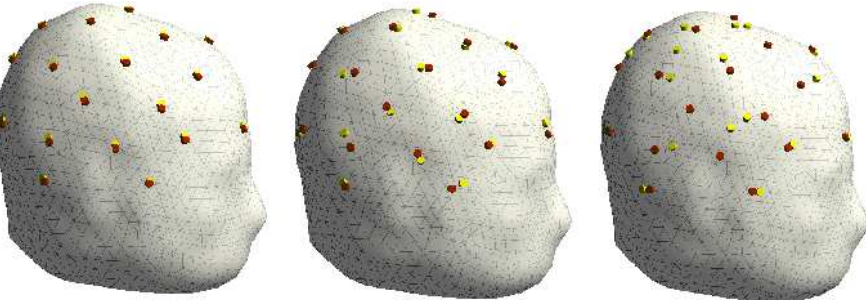

Mean error of $2.02 \mathrm{~mm}$ Mean error of $6.92 \mathrm{~mm}$ Mean error of $11.58 \mathrm{~mm}$

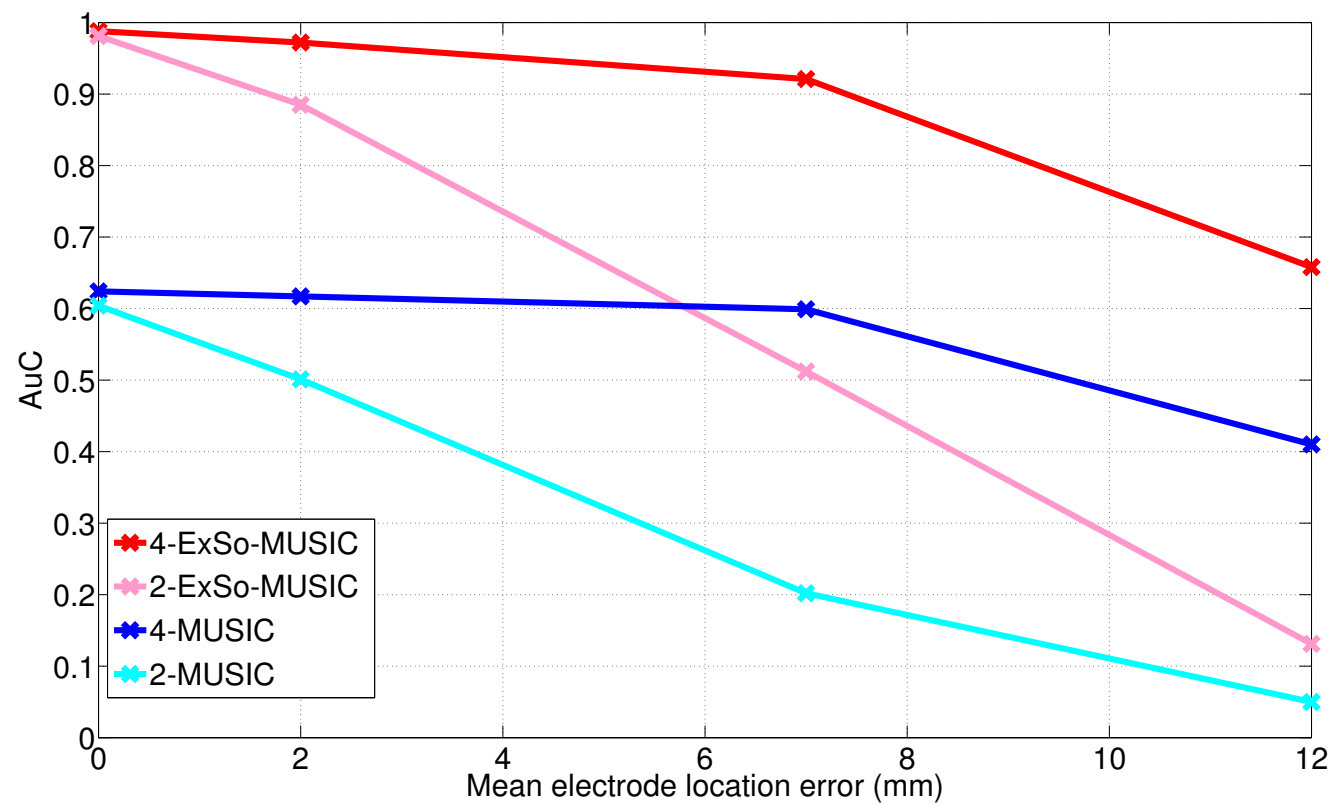

(b)

Figure 9: Performance of the methods with respect to the mean electrode position error. (a) The distances between the original (red) and perturbed (yellow) positions were $0.00 \pm 0.00,2.02 \pm 0.65,6.92 \pm 2.60$ and $11.58 \pm 5.52 \mathrm{~mm}$. Data were simulated from two $1000 \mathrm{~mm}^{2}$ "single-patch" ExSo's located in the frontal and parietal regions. (b) $\mathrm{AuC}$ of the four methods as a function of the mean electrode location error. 
statistics.

The originality of the ExSo principle introduced herein is twofold. Firstly, it relies on the factorization of the multiple dipole activity within the extended source in order to summarize the large number of dipoles into a unique entity and therefore return to an over-determined mixture. This is made possible by the synchronization property of neuronal populations within the source. Indeed, it is very unlikely that uncorrelated or weakly correlated activities within a cortical area could generate signals like epileptic spikes on surface recordings. Indeed, the area and the geometry of the cortical source, along with the synchronisation level of activities within the source are mandatory factors influencing the way cortical activity is reflected at the level of scalp EEG recordings (Ebersole, 1997; Gloor, 1985, 1991). Accordingly, simultaneous scalp and intracranial (ECoG) recordings have clearly illustrated that scalp-recognizable spikes are associated with intracranial events that arise in a highly-correlated manner, i.e. synchronously, with very similar shapes and amplitude, on several ECoG channels. Contrarily, no clear paroxysm emerges from background EEG activity when the synchrony and/or the cortical area are insufficient (see Tao et al., 2005, for illustrative examples).

In the specific context of non-pathological (such as somatosensory evoked field/potentials) signals as well, non parametric localization methods such as the "Time-Coherent Expansion" (David and Garnero, 2002) have exploited uniform current distributions within extended cortical sources. Parametric approaches have also been able to localize such synchronous sources with a small number of equivalent current dipoles (Liu and Schimpf, 2006; Mosher 
and Leahy, 1998). However, these methods took advantage of the high correlation between distant point-like sources and have mostly addressed the question of the interaction across brain areas rather than the geometry of the extended sources. To account for cortical distributed sources, multipolar models (which can be considered as a generalization of the dipolar model) have also been proposed (Jerbi et al., 2002; Nolte and Curio, 1997). One major advantage of the multipolar model is that an extended source of arbitrary current distribution at a given time can be modelled with a relatively few number of parameters. Following this approach, first-order multipolar models proved to be more efficient than classical dipole models to localize the centroid of extended cortical sources (Jerbi et al., 2004). Unfortunately, the source geometry is hardly retrieved from multipolar parameters unless assumptions on the source shape are made and high signal-to-noise ratio in data are considered (Nolte and Curio, 2000).

Therefore in order to recover the exact shape of the source a novel parameterization of the source distribution is described. This constitutes the second novel aspect of the ExSo principle, along with the introduction of an efficient and low-cost algorithm for minimizing the metric. In the context of radiocommunications, Valaee et al. (1995) have introduced the "Distributed Source Parameter Estimator" (DSPE) to estimate the parameters controlling the spatial distribution of the source. However, as is, this method cannot be used in the context of brain source localization of EEG/MEG activities. Indeed, the definition of the complex source topology would require many parameters and thus would involve a costly optimization of the DSPE criterion. To overcome this difficulty and to reduce the high computation complexity 
in the resolution of the inverse problem, we chose to make use of the fact that any extended source can be expressed as the union of elementary geometric surfaces like disks, that are described with two parameters only. In practice, only a finite number of circular patches is sufficient to precisely recover the considered extended source. In other words, the search for an extended source of unknown geometry is transformed into the search for a finite number of elementary surfaces of only two parameters.

The novelty of ExSo-MUSIC also resides in the use of HO statistics. In the context of radiocommunications, the properties of $\mathrm{HO}$ statistics have been successfully exploited in various methods specifically dedicated to the estimation of the Direction-Of-Arrival (DOA) for radio waves (see Chevalier et al., 2009, for a review). In particular, these methods were shown to be less sensitive than SO ones, to perturbations induced by a Gaussian noise with an unknown spatial coherence, to modelling errors, or to a small number of sensors. As previously reported in several studies, background EEG activity can reasonably be considered as Gaussian (Elul, 1969; Knuutila and Hämäläinen, 1987; McEwen and Anderson, 1975). As higher order cumulants of a Gaussian process are null, HO approaches are suited as they are asymptotically insensitive to background EEG activity. Moreover, in real data, background activity is expected to be spatially correlated, this correlation being unknown. Such property penalizes most of SO approaches (such as MUSIC) whereas it is likely to have no impact on HO approaches as the contribution of background activity is suppressed anyways. We recently made use of HO statistics in source localization of EEG/MEG signals extending the concept of deflation source localization (RAP-MUSIC, Mosher 
and Leahy, 1999) to fourth order statistics and showed that their resolution was clearly improved in the case of very close sources, small number of electrodes, or strong background activity (Albera et al., 2008). Similarly, in the present study, the use of HO statistics highly increases the performance of our method, especially when low MSBR's are considered. This is an interesting property in the particular context of epileptic paroxysms, for which the involvement of deep mesial brain sources is common and gives rise to weak amplitude scalp signals compared to the background EEG. Accordingly, in many instances of our simulation study, sources in the cingulate, parahippocampal, or orbitofrontal gyri, as well as in the temporal pole and temporal basal region were retrieved only with the algorithms upgraded to HO statistics.

It is noteworthy that for our simulation study, we assumed that the neuronal populations with highly-correlated activities were contiguous within the extended source. Physiologically other configurations may appear where non-contiguous, yet highly-correlated, populations are involved (distributed sources). This kind of configuration is not unfavourable in our case, since according to our definition, an ExSo is not defined by any spatial constraint but only by the correlation properties of the source activities. Therefore, $2 q$ ExSo-MUSIC is also able to localize an extended source made up of several non-contiguous small groups of dipoles with correlated activities.

In this study, the performance of $2 q$-ExSo-MUSIC was evaluated on simulated data. Indeed, the validation of source localization methods implies that the "ground truth" is known. Unfortunately, this is difficult to achieve when working with real data, and more particularly in the context of partial 
epilepsies, as the exact localisation of the region(s) responsible for interictal spikes or ictal discharges cannot be defined with certainty. At best, it can be approached only when all functional (including invasive recordings) and structural explorations converge towards the same hypothesis. Alternatively, simulations for which both the geometry and the localisation of sources are defined a priori can assist the validation procedure (Grova et al., 2006). In this study, we focused on the realism of our simulations with regard to the source location and geometry. Firstly, our model of EEG generation combines a model of distributed current dipoles and a model of coupled neuronal populations in order to describe as accurately as possible the spatio-temporal properties of sources of brain electrical activity. Secondly, cortical sources of different size were defined in various lobes on a realistic mesh of the cortical surface. These sources were used to generate synthetic data aimed at resembling as much as possible real interictal EEGs recorded in adult partial epilepsies. Thirdly, we considered highly correlated dipole activities within the source to reflect the hypersynchronisation property of the epileptic tissue.

In these situations where the characteristics of the cerebral source is known, our method implemented for $q=2$ clearly showed higher performance, in terms of TPF/FPF ratio, compared to the $2 q$-MUSIC $(q=1,2)$ algorithm. This was observed for almost all considered ExSo sizes and locations, and was particularly marked for signals with low MSBR (i.e. interictal spikes resulting from the activity of ExSo's of small size or located in deep brain regions).

However these simulated results cannot entirely predict the performance of the method in real conditions. More complex simulation scenarios, in 
particular regarding the number of sources, their spatial configuration and the level of synchronisation between neuronal populations as well as the comparison of our approach with other methods more specially designed for the localisation of distributed brain sources are required. This should open a gate towards the application of $2 q$-ExSO-MUSIC to real signals and provide a frame to better interpret results obtained in patients.

\section{Acknowledgement}

This work was supported by a fellowship from the "Region Bretagne" (Program ACOMB, project Solare).

\section{Appendix A. Cumulants and statistical matrix}

The cumulants are quantities that entirely describe the statistical behavior of a random variable. For more theoritical informations see (McCullagh, 1987). In practice, the cumulants are computed from $K$ available samples of the data $\boldsymbol{x}(t)$ ordered in the $(N \times K)$ spatio-temporal matrix $\boldsymbol{X}$. The covariance and quadricovarince matrices of the data are estimated by $\hat{\boldsymbol{C}}_{2, \boldsymbol{x}}=\boldsymbol{X} \boldsymbol{X}^{\top} / K$ and

$$
\begin{aligned}
\hat{\boldsymbol{C}}_{4, \boldsymbol{x}}= & (\boldsymbol{X} \oslash \boldsymbol{X})(\boldsymbol{X} \oslash \boldsymbol{X})^{\top} / K-\left(\boldsymbol{X} \boldsymbol{X}^{\top}\right) \otimes\left(\boldsymbol{X} \boldsymbol{X}^{\top}\right) / K^{2} \\
& -(\boldsymbol{X} \oslash \boldsymbol{X}) \mathbf{1}_{N^{2}} \mathbf{1}_{N^{2}}^{\top}(\boldsymbol{X} \oslash \boldsymbol{X})^{\top} / K^{2} \\
& -\left[\left(\boldsymbol{X} \otimes \mathbf{1}_{N}\right)\left(\mathbf{1}_{N} \otimes \boldsymbol{X}\right)^{\top}\right] \odot\left[\left(\mathbf{1}_{N} \otimes \boldsymbol{X}\right)\left(\boldsymbol{X} \otimes \mathbf{1}_{N}\right)^{\top}\right] / K^{2}
\end{aligned}
$$

, respectively, where $\oslash$ is the Khatri-Rao product operator (also refered as column-wise Kronecker product), $\odot$ is the Hadamard product operator and $\mathbf{1}_{N}$ is a $N$-dimensional vector of ones. The Khatri-Rao product $\boldsymbol{X} \oslash \boldsymbol{X}$, also 
known as column-wise Kronecker product, is equal to $\left[\boldsymbol{x}_{1} \otimes \boldsymbol{x}_{1}, \boldsymbol{x}_{2} \otimes \boldsymbol{x}_{2}, \ldots, \boldsymbol{x}_{K} \otimes\right.$ $\boldsymbol{x}_{K}$ ] where $\boldsymbol{x}_{k}$ is the $k$ th column of $\boldsymbol{X}$. The Hadamard product is a term by term matricial product.

\section{Appendix B. Validity of the "ExSo" principle}

The validity of our approach can be proved in a probabilistic sense. It is based on both following propositions:

P1. For every $\Theta$, it exists at least one threshold $\lambda_{1}$ such that for every $\lambda$ smaller than or equal to $\lambda_{1}$, the estimate $\hat{\Theta}^{2 q-\text { ExSo-MUSIC }}(\lambda)$ is a subset of $\Theta$.

P2. For every $\Theta$, it exists at least one threshold $\lambda_{2}$ such that for every $\lambda$ bigger than or equal to $\lambda_{2}$ the estimate $\hat{\Theta}^{2 q-\text { ExSo-MUSIC }}(\lambda)$ entirely recovers $\Theta$.

Let's verify that proposition P1) is almost surely true in our EEG/MEG localization context. With this aim, we introduce the probability, as a function of $\lambda$, that $\hat{\Theta}^{2 q-\text { ExSo-MUSic }}(\lambda)$ is a subset of $\Theta$, say $\mathrm{P}\left(\hat{\Theta}^{2 q-\text { ExSo-Music }}(\lambda) \subseteq \Theta\right)$. If it exists a threshold $\lambda_{1}$ such that for all $\lambda \leq \lambda_{1}, \operatorname{P}\left(\hat{\Theta}^{2 q \text {-ExSo-MUsic }}(\lambda) \subseteq \Theta\right)$ is zero, then proposition P1) will be almost surely true. The probability $\mathrm{P}\left(\hat{\Theta}^{2 q \text {-ExSo-MUSIC }}(\lambda) \subseteq \Theta\right)$ was estimated from 27 ExSo's $\Theta$ of 315,500 and 1000 $\mathrm{mm}^{2}$ and the result is shown in figure B.10 for $q=1,2$. It appears that this estimated probability reached 0 beyond $\lambda=0.03$ for $q=1$ and $\lambda=0.05$ for $q=2$, hence the result.

As far as proposition P2) is concerned, it is true because $\hat{\Theta}^{2 q-\text { ExSo-MUSIC }}(1)$ recovers all the cortex and consequently entirely $\Theta$. Obviously, this value 


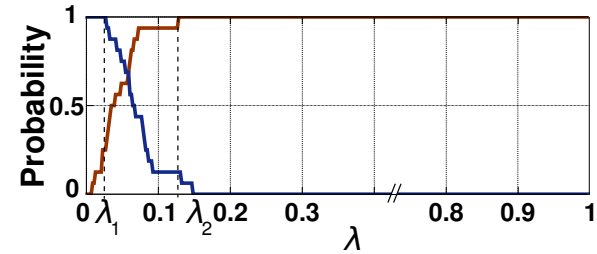

(a) second order

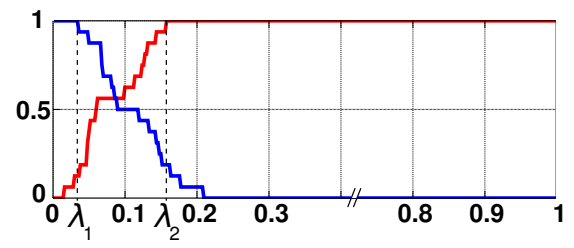

(b) fourth order

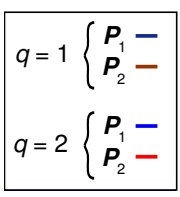

Figure B.10: Probability of the estimated extended source $\hat{\Theta}^{2 q \text {-ExSo-MUSIC }}(\lambda)(q=1,2)$ to be a subset of the actual extended source $\Theta$ (referred as $\boldsymbol{P}_{1}$ ) and probability of $\hat{\Theta}^{2 q-\operatorname{ExS} \text {-MUSIC }}(\lambda)$ to entirely recover $\Theta\left(\right.$ referred as $\left.\boldsymbol{P}_{2}\right)$.

of $\lambda_{2}$ is not satisfying. Therefore, we show in figure B.10, for $q=1,2$, that proposition P2) is almost surely true for $\lambda_{2}$ much less than $1\left(\lambda_{2}=0.12\right.$ for $q=1, \lambda_{2}=0.16$ for $q=2$ ) by representing the estimate of the probability $\mathrm{P}\left(\hat{\Theta}^{2 q-\text { ExSo-MUSIC }}(\lambda) \cap \Theta=\Theta\right)$ from the same 27 ExSo's $\Theta$. Interestingly, this probability is equal to one for $\lambda$ closer to $\lambda_{1}$ than 1 .

\section{References}

Albera, L., Ferréol, A., Cosandier-Rimele, D., Merlet, I., Wendling, F., February 2008. Brain source localization using a fourth-order deflation scheme. IEEE Trans Biomed Eng 55 (2), 490-501.

Albera, L., Kachenoura, A., Wendling, F., Senhadji, L., Merlet, I., 2010. A joint use of second, third and fourth order statistics for independent component analysis of biomedical signals. In: EMBC 10, 32nd Annual International Conference of the IEEE Engineering in Medicine and Biology Society, Buenos Aires, Argentina, August 31 - September 4 2010. In press.

Birot, G., Albera, L., Chevalier, P., August 2010. Sequential high resolution 
direction finding from higher order statistics. IEEE Trans Signal Process 58 (8), 4144-4155.

Chevalier, P., Ferréol, A., Albera, L., August 2006. High-resolution direction finding from higher order statistics: The $2 q$-MUSIC algorithm. IEEE Trans Signal Process 54 (8), 2986-2997.

Chevalier, P., Ferréol, A., Albera, L., 2009. High-resolution DOA estimation with higher order statistics. In: Tuncer, T. E., Friedlander, B. (Eds.), Classical and Modern Direction-of-Arrival Estimation. Academic Press.

Chevalier, P., Ferréol, A., Albera, L., Birot, G., November 2007. Higher order direction finding from arrays with diversely polarized antennas: The PD-2q-MUSIC algorithms. IEEE Trans Signal Process 55 (11), 5337-5350.

Congedo, M., Gouy-Pailler, C., Jutten, C., June 2008. On the blind source separation of human electroencephalogram by approximate joint diagonalization of second order statistics. Clin Neurophysiol 119 (12), 2677-2686.

Cosandier-Rimélé, D., Badier, J., Chauvel, P., Wendling, F., March 2007. A physiologically plausible spatio-temporal model for depth-EEG signals recorded with intracerebral electrodes in human partial epilepsy. IEEE Trans Biomed Eng 54 (3), 380-388.

Cosandier-Rimélé, D., Merlet, I., Badier, J., Chauvel, P., Wendling, F., April 2008. The neuronal sources of EEG: Modeling of simultaneous scalp and intracerebral recordings in epilepsy. Neuroimage 42 (1), 135-146.

David, O., Garnero, L., November 2002. Time-coherent expansion of MEG/EEG cortical sources. Neuroimage 17 (3), 1277-1289. 
de Munck, J., Bijma, F., September 2009. Three-way matrix analysis, the MUSIC algorithm and the coupled dipole model. J Neurosci Methods $183(1), 63-71$.

Ebersole, J. S., 1997. Magnetoencephalography/magnetic source imaging in the assessment of patients with epilepsy. Epilepsia 38 (Suppl 4), S1-5.

Ebersole, J. S., 2000. Noninvasive localization of epileptogenic foci by EEG source modeling. Epilepsia 41 (Suppl 3), S24-33.

Elul, R., April 1969. Gaussian behavior of the electroencephalogram: changes during performance of mental task. Science 164 (3877), 328-331.

Funke, M., Constantino, T., Van Orman, C., Rodin, E., October 2009. Magnetoencephalography and magnetic source imaging in epilepsy. Clin EEG Neurosci 40 (4), 271-280.

Gavaret, M., Badier, J.-M., Marquis, P., McGonigal, A., Bartolomei, F., Regis, J., Chauvel, P., August 2006. Electric source imaging in frontal lobe epilepsy. J Clin Neurophysiol 23 (4), 358-370.

Gloor, P., October 1985. Neuronal generators and the problem of localization in electroencephalography: application of volume conductor theory to electroencephalography. J Clin Neurophysiol 2 (4), 327-354.

Gloor, P., November 1991. Preoperative electroencephalographic investigation in temporal lobe epilepsy: extracranial and intracranial recordings. Can J Neurol Sci 18 (Suppl 4), 554-558, review. 
Gonçalves, S., de Munck, J., Verbunt, J., Bijma, F., Heethaar, R., Lopes da Silva, F., September 2003. In vivo measurement of the brain and skull resistivities using an EIT-based method and realistic models for the head. IEEE Trans Biomed Eng 50 (9), 1124-1128.

Grech, R., Cassar, T., Muscat, J., Camilleri, K. P., Fabri, S. G., Zervakis, M., Xanthopoulos, P., Sakkalis, V., Vanrumste, B., November 2008. Review on solving the inverse problem in EEG source analysis. J Neuroeng Rehabil $7,5-25$, review.

Grova, C., Daunizeau, J., Lina, J. M., Benali, H., Gotman, J., February 2006. Evaluation of EEG localization methods using realistic simulations of interictal spikes. Neuroimage 29 (3), 734-753.

James, C. J., Hesse, C. W., February 2005. Independent component analysis for biomedical signals. Physiol Meas 26 (1), R15-R39.

Jerbi, K., Baillet, S., Mosher, J., Nolte, G., Garnero, L., Leahy, R., June 2004. Localization of realistic cortical activity in MEG using current multipoles. Neuroimage 22 (2), 779-793.

Jerbi, K., Mosher, J., Baillet, S., Leahy, R., February 2002. On MEG forward modelling using multipolar expansions. Phys Med Biol 47 (4), 523-555.

Kaveh, M., Barabell, A., April 1986. The statistical performance of the MUSIC and the minimum norm algorithms in resolving plane waves in noise. IEEE Transactions On Aerospace and Electronic Systems 34, 331-341.

Knuutila, J., Hämäläinen, M. S., 1987. Characterization of brain noise using 
a high sensitivity 7-channel magnetometer. In: Atsumi, K. (Ed.), Proc. 6th Int. Conf. Biomag. Eds., Tokyo, Japan, pp. 186-189.

Küçükaltun-Yildirim, E., Pantazis, D., Leahy, R., September 2006. Taskbased comparison of inverse methods in magnetoencephalography. IEEE Trans Biomed Eng 53 (9), 1783-1793.

Liu, H., Schimpf, P., April 2006. Efficient localization of synchronous EEG source activities using a modified RAP-MUSIC algorithm. IEEE Trans Biomed Eng 53 (4), 652-661.

McCullagh, P., 1987. Tensor Methods in Statistics. Chapman and Hall, Monographs on Statistics and Applied Probability.

McEwen, J., Anderson, G., September 1975. Modeling the stationarity and gaussianity of spontaneous electroencephalographic activity. IEEE Trans Biomed Eng 22 (5), 361-369.

Merlet, I., July 2001. Dipole modeling of interictal and ictal EEG and MEG paroxysms. Epileptic Disord Special Issue, 11-36.

Merlet, I., Gotman, J., June 1999. Reliability of dipole models of epileptic spikes. Clin Neurophysiol 110 (6), 1013-1028.

Metz, C., 1986. Roc methodology in radiologic imaging. Invest Radiol 21 (9), $720-732$.

Michel, C. M., Murray, M. M., Lantz, G., Gonzalez, S., Spinelli, L., de Peralta, R. G., October 2004. EEG source imaging. Clin Neurophysiol 115 (10), 2195-2222. 
Mosher, J. C., Leahy, R. M., November 1998. Recursive MUSIC: A framework for EEG and MEG source localization. IEEE Trans Biomed Eng 45 (11), $1342-1354$.

Mosher, J. C., Leahy, R. M., February 1999. Source localization using Recursively Applied and Projected (RAP) MUSIC. IEEE Trans Signal Process 47 (2), 332-340.

Mosher, J. C., Lewis, P. S., Leahy, R. M., June 1992. Multiple dipole modeling and localization from spatio-temporal MEG data. IEEE Trans Biomed Eng 39, 541-557.

Nolte, G., Curio, G., September 1997. On the calculation of magnetic fields based on multipole modeling of focal biological current sources. Biophys J 73 (3), 1253-1262.

Nolte, G., Curio, G., Oct. 2000. Current multipole expansion to estimate lateral extent of neuronal activity: a theoretical analysis. IEEE Trans Biomed Eng 47 (10), 1347-1355.

Plummer, C., Harvey, A. S., Cook, M., February 2008. EEG source localization in focal epilepsy: where are we now? Epilepsia 49 (2), 201-218.

Plummer, C., Wagner, M., Fuchs, M., Vogrin, S., Litewka, L., Farish, S., Bailey, C., Harvey, A., Cook, M., October 2010. Clinical utility of distributed source modelling of interictal scalp EEG in focal epilepsy. Clin Neurophysiol 121 (10), 1726-1739.

Porat, B., Friedlander, B., April 1988. Analysis of the asymptotic relative efficiency of the MUSIC algorithm. IEEE Trans Acoust 36 (4), 532-544. 
Schmidt, R. O., March 1986. Multiple emitter location and signal parameter estimation. IEEE Trans Antennas Propag 34 (3), 276-280, reprint of the original 1979 paper from the RADC Spectrum Estimation Workshop.

Stoica, P., Nehorai, A., May 1989. MUSIC, Maximum Likelihood and Cramer Rao Bound. IEEE Transactions On Acoustics, Speech, Signal Processing 37 (12), 720-741.

Stoica, P., Nehorai, A., December 1990. Maximum likelihood and cramer rao bound: Further results and comparisons. IEEE Transactions On Acoustics, Speech, Signal Processing 38 (12), 2140-2150.

Tao, J., Hawes-Ebersole, S., Ebersole, J., May 2005. Intracranial EEG substrates of scalp EEG interictal spikes. Epilepsia 46 (5), 669-676.

Valaee, S., Champagne, B., Kabal, P., September 1995. Parametric localization of distributed sources. IEEE Trans Signal Process 43 (9), 2144-2153.

Verhellen, E., Boon, P., September 2007. EEG source localization of the epileptogenic focus in patients with refractory temporal lobe epilepsy, dipole modelling revisited. Acta neuroligica Belgia 107 (3), 71-77, review.

Vigario, R., Oja, E., 2008. BSS and ICA in neuroinformatics: From current practices to open challenges. IEEE Rev Biomed Eng 1, 50-61.

Wendling, F., Bellanger, J., Bartolomei, F., Chauvel, P., October 2000. Relevance of nonlinear lumped-parameter models in the analysis of depth-EEG epileptic signals. Biol Cybern 83 (4), 367-378. 


\section{Figure and table captions}

Figure 1

(a) Schematic representation of a cortex exhibiting a region of epileptic activity $\Theta$ (in gray). The entire cortex (including gray regions) generates also background activity. (b) An example of ExSo's configuration. On this schematic representation, the cortical area $\Theta$ exhibiting epileptic activity can be partitioned in two extended sources $\theta_{1}$ (in green) and $\theta_{2}$ (in red). In $\theta_{1}$, two spatially distinct patches are considered. The activity within and between the two patches are synchronized. Therefore $\theta_{1}$ will be considered as a single ExSo. $\theta_{2}$ is made of a single patch. The activity within this patch is synchronized but is independent from the activity in $\theta_{1}$. Therefore $\theta_{2}$ is considered as another ExSo.

Figure 2

The ExSo-MUSIC principle. (a) Schematic representation of the ExSoMUSIC principle. When the threshold $\lambda$ increases, the number of concatenated circular sets $\tilde{\theta}$ increases, enlarging the surface of the estimated extended source. (b) Example of result using 4-ExSo-MUSIC method. The actual extended source $\Theta$ is red. The true estimated parts $\hat{\Theta}^{4 \text {-ExSo-MUSIC }}(\lambda) \cap \Theta$ are beige, and the false estimated parts $\hat{\Theta}^{4-E x S o-M U S I C}(\lambda)-\hat{\Theta}^{4-E x \text { So-MUSIC }}(\lambda) \cap \Theta$ are blue. In practice, as the source space is a triangular mesh of the cortical surface, we cannot construct perfectly circular disks. Instead we consider pseudo-disks, made of one or several triangles and parameterized by the disk area and the position of its center, as defined by the position of the germ triangle. For 
a mesh with sufficiently high resolution, any ExSo can be approximated by the union of these pseudo-disks.

\section{Figure 3}

Simulation of scalp EEG spikes. (a) An extended source of 1000 triangles $\left(\approx 10 \mathrm{~cm}^{2}\right)$ was manually defined on a mesh of the cortical surface using Paraview (Paraview, Kitware Inc., NY, US). Dipoles are placed all over the mesh at the barycenter of each triangle and oriented orthogonal to the triangle surface. Within the source, each dipole is assigned with a spike-like activity. For the whole source, a series of 1000 highly synchronized activities is generated using the neuronal population model (50 are displayed here). In the remaining part of the mesh dipoles are assigned with uncorrelated background activity. (b) Using this source configuration, a realistic head model and a given set of 31 electrodes, EEG data were computed solving numerically the forward problem. In this particular case, epileptic spikes generated from the left superior frontal ExSo are clearly identified on F3, FC1 and Fz.

\section{Figure 4}

Performance of the source localization approaches with respect to the MSBR. (a) In this study, data were simulated from one "single-patch" ExSo of $1000 \mathrm{~mm}^{2}$ placed in the left lateral occipital region. Six different values of MSBR (from 18.3 to $3.3 \mathrm{~dB}$ ) are illustrated. (b) For the $2 q$-ExSo-MUSIC and $2 q$-MUSIC $(q=1,2)$ methods, the Area under the ROC Curve (AuC) is plotted as a function of the MSBR. 
Figure 5

Performance of the source localization approaches with respect to the area of the ExSo. (a) In this study, data were simulated from one "singlepatch" ExSo located in the left superior frontal region. The source area varied from 50 to $2000 \mathrm{~mm}^{2}$. Four different areas (50, 200, 1000 and $2000 \mathrm{~mm}^{2}$ ) are illustrated. (b) AuC of the $2 q$-ExSo-MUSIC and $2 q$-MUSIC $(q=1,2)$ methods as a fonction of the ExSo area. The MSBR is also reported as it is relied to the ExSo area.

Figure 6

Performance of the source localization approaches with respect to the location of the ExSo. (a) In this study nine different source locations and three different areas $\left(315,500\right.$ and $\left.1000 \mathrm{~mm}^{2}\right)$ were used to simulate the data. The nine source locations are illustrated for an ExSo of $500 \mathrm{~mm}^{2}$. (b) $\mathrm{AuC}$ of the $2 q$-ExSo-MUSIC and $2 q$-MUSIC $(q=1,2)$ methods as a fonction of the ExSo location and for ExSo area of 315, 500 and $1000 \mathrm{~mm}^{2}$.

\section{Figure 7}

Examples of localization results of 4-ExSo-MUSIC, 2-ExSo-MUSIC, 4MUSIC and 2-MUSIC. The threshold was choosen such that $\overline{\mathrm{FPF}} \approx 5.10^{-3}$ (this corresponds to an area of about $350 \mathrm{~mm}^{2}$ ). (a) $1000 \mathrm{~mm}^{2}$ ExSo in the left pole. (b) $1000 \mathrm{~mm}^{2}$ ExSo in the left frontodorsal gyrus. (c) $1000 \mathrm{~mm}^{2}$ ExSo in the left parahippocampus gyrus. $\left(^{*}\right)$ Misestimated parts are located in the cingulate region. 
Figure 8

Localization results of the $2 q$-ExSo-MUSIC and $2 q$-MUSIC $(q=1,2)$ methods in the context of three multi-patch configurations. (a) two "singlepatch" ExSo's (i.e. with interpatch independent activity) located in the superior and middle temporal gyrus. (b) one "double-patch" ExSo (i.e. with interpatch synchronized activity). (c) one "double-patch" ExSo (i.e. with interpatch synchronized activity) and one "single-patch" ExSo (i.e. with activity independent from the first two) located in the middle frontal gyrus.

Figure 9

Performance of the methods with respect to the mean electrode position error. (a) The distances between the original (red) and perturbed (yellow) positions were $0.00 \pm 0.00,2.02 \pm 0.65,6.92 \pm 2.60$ and $11.58 \pm 5.52 \mathrm{~mm}$. Data were simulated from two $1000 \mathrm{~mm}^{2}$ "single-patch" ExSo's located in the frontal and parietal regions. (b) $\mathrm{AuC}$ of the four methods as a function of the mean electrode location error.

Figure B.10

Probability of the estimated extended source $\hat{\Theta}^{2 q \text {-ExSo-MUsic }}(\lambda)(q=1,2)$ to be a subset of the actual extended source $\Theta$ (referred as $\boldsymbol{P}_{1}$ ) and probability of $\hat{\Theta}^{2 q-\text { ExSo-MUSIC }}(\lambda)$ to entirely recover $\Theta$ (referred as $\boldsymbol{P}_{2}$ ).

\section{Table 1}

$\mathrm{AuC}$ for all four localization methods obtained in the context of three multipatch ExSo configurations. 